PACS :12.19. $-g ; 12.90 .+b$

\title{
FORMALIZATION OF COGNITION PROCESS AS AN ADDITIONAL COMPONENT RESPONSIBLE FOR DEVELOPMENT OF THEORETICAL PHYSICS
}

\author{
Jarosław Kaczmarek \\ Institute of Fluid-Flow Machinery, Polish Academy of Sciences \\ 80-231 Gdańsk, ul. J.Fiszera 14, Poland \\ E-mail: jarekk@imp.gda.pl
}

Received December 21, 2018; accepted 22 January 2019

Theoretical physics has attained stage when new methodological approaches should be taken into considerations. In particular they should introduce larger discipline in theoretical speculations. In this paper one introduces concept of cognition manifold as methodological framework for development of description of reality by theoretical physics with the aid of theoretical speculations. One assumes that this is a way for constructing fundamental and universal physical theories. The cognition manifold is composed of basis which represents models of our space as a medium and fibres representing models of processes in corresponding space. Models are based on accessible experimental results and also on new assumptions and hypotheses obtained by theoretical speculations. In order to maintain dyscipline in these speculations one considers status of assumptions and theories spanned on cognition manifold as a function defined on elements of fibres. One accentuates importance of selforganizing reasoning as more appropriate for recognition of reality in comparison with precise pure mathematical proof. One considers also proof with respect to reality. This paper is considered as an attempt to formalization of cognition process what is manifested by three main components: cognition manifold, status of assumptions and theories and concept of selforganizing reasoning.

KEYWORDS: methodology of constructing universal physical theories, cognition manifold, formalization of cognition process

\section{Introduction}

Contemporary theoretical physics manifests various attempts to description of reality. However, no clear methodology for doing this is elaborated. On the one hand one applies mathematics with precise proofs neglecting analysis to what degree this mathematics reflects reality. On the other hand one constructs many simplified models which reflect directly experimental results.

In both cases we should not be satisfied. Mathematics is a system placed on the inside of our brain and as such is not associated directly with reality. Simplified models which are constructed in accordance with chosen set of experimental results lead to theories which are not universal.

We try in theoretical physics to construct fundamental and universal theory as far as it is possible. This is so since universal theory is real success for our civilization. By means of universal theory we can predict many phenomena as well as to develop various applications.

One suggests that theoretical physics should consider two aspects: the first one is related to correct application of mathematics within physical theory and the second one is related to quality of fitting of the applied mathematical theory to reality.

Various methodologies are suggested within philosophy of physics. Let us mention rejection of theories by falsification [3]. This method is usually too radical since approximation of reality by theory is usually not entirely perfect. Then corresponding rejection could be associated with loss of context for further development of theoretical description. As a result of this development of theoretical physics could be stopped.

On the other hand introduction of a paradigm [4] allows us to continue development until more complete recognition of theory within this paradigm is obtained. Then, development of theoretical physics is more fluent. However rejection of paradigm with time seems also be too radical since (C) Jaroslaw Kaczmarek, 2019 
correctnees of approximation of reality by theories are not usually entirely investigated. Let us mention range of validity of theory with respect to correctness of approximation of reality. This aspect is usually not investigated. We would say that each theory which had partial success approximates reality to some degree. Therefore such a theory should not be entirely rejected. Instead of this placement of theory with respect to its quality should be better determined taking into account especially its range of validity.

Let us note that fitting of theory to reality is usually estimated by accordance of theoretical results with experimental results. However, when we come to more fundamental theories usually related to smaller scales then conjugation of interpretation of experimental results and theory increases. We are not able to carry our precisely experiments in small scale and related to chosen particular phenomenon. We are usually forced to decipher obtained experimental results from more complex processes modelled within a given theory. Thereby, accordance of theoretical results and experimental results as well as interpretation of obtained experimental results depend on theory applied.

Let us consider an example. We observe that system of cosmological objects expands. It means that in the past all they should be close one to another. This leads to concept of Big Bang. Something small exploded and as a result of this we have to do with expanding system of astronomical objects. One assumes in theories that this explosion was associated with explosion of space and corresponding inflation associated with creation of pairs of particles and antiparticles. These particles, owing to unknown asymmetry, create contemporary matter.

On the other hand we can assume that we can admit explosion of space in very dense matter on the inside of giant black hole. Then we have to explain why such explosion of space could happen. This is possible when we neglect model of point-like particle. Then annihilation of electron and positron is associated with emission of electromagnetic radiation and also joining of components of electron and positron what leads to explosion of space in place of annihilation. Such a model of explosion of space and corresponding Big Bang model is based on three-positron structure of proton and is discussed in [16]. Within this model we understand what was exploded and why.

We frequently encounter interpretation of experimental results by one chosen theory. Let us mention that one suggests that neutrina have mass. This is interpretation obtained with the aid of the standard model and related to experimental observation that neutrina change their type with time during their motion. However we can admit the situation when neutrina can change their type and have mass equal to zero when we apply the vacuum medium mechanics [17].

We see that the same experimenal results on expansion of system of astronomical objects and evolution of type of neutrina can lead to entirely different theoretical interpretations. Therefore we have to consider whether our methodology of modelling of reality is appropriate and whether we can improve this methodology.

By above comments we see that theoretical speculations in relation to physical reality have too large freedom. This is manifested by fact that considerable number of theories can leed to the same results as experimental observations having in mind also quantitative accordance of these results with teoretical prediction. The question is how we can reduce this excessive freedom in creation of theoretical models.

It seems that development of theoretical physics should be supported by formalization of cognition process. This is so since theoretical physics acts on front of cognition in order to recognize reality better. However our possibilities of recognition of reality are connected with our biology. In particular our brain is created in order to improve our cognition. On the other hand, it is not clear to what degree our brain is the only tool for recognition of reality by formal theories. Perhaps we are not able to identify other kinds of perception which take part in supporting of power of our cognition. Perhaps our emotions considered as a driven force for cognition process can be associated with not well understood forms of perception and will be difficult to formalization within a theory.

However taking into account all possible sources of cognition we cannot accept attitude associated with relinquishment of formalization of cognition process. This formalization should be continued as far as it is possible and should lead to placing of other possible mechanisms of cognition more precisely.

In order to provide context for discussion of quality of description of reality by theory one discusses 
in [1], [2] ways describing how theory could appear in our world. To this end one introduces there evolving dynamical system within which logic and mathematics could appear as a result of interactions with external world. Important aspect of this approach is associated with interpretation of implication as related to action of the dynamical system in environment. As a result of this one introduces the notion "status" of assumptions applied to construction of theory and "status" of theory as property which is responsible for quality of approximation of reality by this theory. Consequently the notion of status joins our mathematics considered as internal system in our brain with external world. This in turn allow us to estimate criteria for determination which assumptions and theories are better fitting to reality in comparison with other ones.

Whole methodology based on formalization of cognition process was elaborated during formulation of vacuum medium mechanics. This theory is aimed at satisfying contemporary requirements for fundamental and universal theory. These requirements are related especially to description of dynamics for the smallest scale applied in modelling. This in turn is of key importance for determination of mechanisms of biological evolution or source of precission for theoretical nanotechnology.

Contemporary theories are based predominantly of geometry and symmetries and dynamics of processes on the smallest scales are seen as secondary importance. Furtheremore scale of averaging applied in these theories are not well formalized. As a result of this various interpretations of experimental results are done for reality which is not seen by corresponding theories. Let us mention in this place the Higgs particle. Existence of particle in this place is really detected. However, this elementary particle is not observed directly. Instead of this one observes products of its decay. On the other hand this particle is produced during collisions of protons. It means that it is created directly near particles. However the standard model as quantum theory which replaces elementary particles by waves does not see processes directly near particles. Therefore the standard model is not appropriate theory for interpretation whether detected paricle is the Higgs boson with complicated functions assignet to it.

We see in this place overinterpretation obtained with the aid of the standard model. All this justifies necessity of formalization of cognition process which should be seen as supporting construction of fundamental and universal theories.

\section{A framework for formalization of cognition process based on dynamical systems}

\subsection{On emerging of mathematics within evolving dynamical systems}

We discuss here some results from [1], [2] in order to provide sufficiently large context for discussion of formalization of cognition process.

We assume that our brain and whole biological organism is a dynamical system which can evolve. Thereby, we should look for context for discussion of quality of cognition process by theory based on dynamical systems.

We introduce here dynamical system which can undergo evolution and is able to generate cognition process. Let us assume namely that our dynamical system contains the following subsystems: the neural network dynamical system (N), motor system (M), sensor system (S), environmental dynamical system $(\mathrm{E})$ and the main dynamical system $(\mathcal{M})$.

All introduced subsystems interact by assumption. Source of activity follows from the main dynamical system. It can interact with environmental dynamical system immediately or by means of neural network, motor and sensor systems.

We distinguish the following groups of variables of our dynamical system: $x_{E}, x_{N}, x_{M}, x_{S}, x_{\mathcal{M}}$. In particular, for description of interactions we distinguish subgroups of variables which take part in these interactions directly. They are specified within the following dynamical systems

$$
\dot{x}_{E}=A_{E}\left(\bar{x}_{E}, x_{E M}, x_{E S}, x_{E \mathcal{M}} ; x_{M E}, x_{S E}, x_{\mathcal{M} E}\right) \text {, }
$$




$$
\begin{gathered}
\dot{x}_{M}=A_{M}\left(\bar{x}_{M}, x_{M E}, x_{M N} ; x_{E M}, x_{N M}\right), \\
\dot{x}_{S}=A_{S}\left(\bar{x}_{S}, x_{S E}, x_{S N} ; x_{E S}, x_{N S}\right), \\
\dot{x}_{N}=A_{N}\left(\bar{x}_{N}, x_{N M}, x_{N S}, x_{N \mathcal{M}} ; x_{M N}, x_{S N}, x_{\mathcal{M N}}\right), \\
\dot{x}_{\mathcal{M}}=A_{\mathcal{M}}\left(\bar{x}_{\mathcal{M}}, x_{\mathcal{M E}}, x_{\mathcal{M N}} ; x_{E \mathcal{M}}, x_{N \mathcal{M}}\right) .
\end{gathered}
$$

Particular groups of variables specify parts of subsystems interacting. We discuss the notation for the first subsystem as example, where $x_{E}=\left\{\bar{x}_{E}, x_{E M}, x_{E S}, x_{E \mathcal{M}}\right\}$. Variables $\left\{x_{E M}, x_{E S}, x_{E \mathcal{M}}\right\}$ are designed to describe interaction with other subsystems. On the other hand $x_{M E}, x_{S E}, x_{\mathcal{M E}}$ represent subgroups of variables of remaining subsystems within the first one also in order to describe corresponding interactions.

We do not specify here how such a dynamical system evolves. This is not necessary for our considerations at this moment. However we accentuate this fact since it is important for future investigations on this line as well as for further discussion. Consequently, we introduce here an abstraction level for our considerations. Particular determination of the dynamical system could be seen as entirely separate problem and perhaps even branch of knowledge. This could be asociated with considerations related to biology. However, corresponding determination of the dynamical system can also have entirely abstract form and can be considered within pure mathematics.

Subsystems are designed to describe particular properties of the whole system. Consequently, the external dynamical system describes behaviour of an environment in which the sybsystem $S_{A}=$ $S_{\mathcal{M}} \cup S_{N} \cup S_{M} \cup S_{S}$ acts. Driven force for such an action is generated by the subsystem $S_{\mathcal{M}}$. It can interact with the environment directly or realize an action by means of neural network system and motor and sensory system.

Concept of subsystems suggest that $S_{\mathcal{M}}$ represents the highest level of complexity of an autonomous system which can be interpreted as a living organism for instance. It has to survive in the environment. Therefore this system generates an action. In particular this action is associated with an attractor defined within the main dynamical system.

Whole system $S_{T}=S_{E} \cup S_{\mathcal{M}} \cup S_{N} \cup S_{M} \cup S_{S}$ is called here the total system. Then, $S_{E}$ stand for environment dynamical system and $S_{A}$ is called here the autonomic dynamical system.

Introduced above dynamical system is intended to describe a kind of brain surrounded with some functional subsystems in an abstract form. In particular, having in mind such a concept, the neural network system is introduced.

Logic appears when we have at our disposal a system of formulas and ability to determination of the true. In order to construct the system of formulas we have first to define terms [22].

Terms have to be considered here within the neural network system. We assume that they are associated with action of motor and sensor systems by means of the functions $\pi_{S N}$ and $\pi_{M N}$. They transform the set of variables of the sensory system $V_{S}$ and motor system $V_{M}$ into set of variables of the neural network system $V_{N}$. Thus we have $\pi_{S N}: V_{S} \rightarrow V_{N}$ and $\pi_{M N}: V_{M} \rightarrow V_{N}$. Both functions are determined by means of previously introduced dynamical system (1)-(5).

The set of terms induced by dynamical systems $S_{M}, S_{S}$ is defined by

$$
T=\pi_{M N}\left(V_{M}\right) \cup \pi_{S N}\left(V_{S}\right)
$$

The set $T$ contains variables associated with motor and sensor systems expressed within the neural network system.

When we construct theory then the set of terms $T$ is contained in the set of formulas $F[22]$. Consequently, the question is how we define next elements of the set $F$. In order to do it we should have at our disposal relations as well as possibility of taking conjunction, alternative, negation and 
implication. They should be expressed within the neural network. Therefore we should discuss a way on which such notions could happen in the neural network.

Let us notice that conjunction, alternative and negation seem to be relatively simple in order to express them in the neural network. The word "and" is easily understand as a primary notion. Negation appears also in a natural way as opposition to "satisfying a relation". Alternative has a similar level of complexity.

Implication seems to be more complicated and also more important for logic. Its role in proving of the true is crucial. Furthermore, it is not evident directly how to introduce implication. In classical logic the implication is introduced formally by assumed way of valuation. However, intuition associated with implication suggests a causality. This in turn suggests that implication is not entirely internal notion related to the neural network system. It should be associated with processes in the environment induced by the dynamical system $S_{A}$ in order to reflect the causality. In this case environment play role of real world. Thereby, we should postulate a way for introduction of implication in our dynamical system.

We admit in general evolution of introduced subsystems. In particular this is the case for the neural network dynamical system. At this moment we do not discuss particular mechanisms of such an evolution. We expect in general that development attained by this evolution leads to possibility of generation of relations, formulas and methods of valuation of sentences. In particular relations could appear as a result of action of the main dynamical system which will be discussed below.

We decide which mechanism of evolution is admitted into considerations. Thereby, we discuss then an abstract system which could generate logic and a kind of thinking. Consequently, using such a way of modelling we should be motivated by concepts of phenomena which we would like to obtain as properties of our dynamical system. Let us discuss some properties related to logic which could appear during modelling of a reality.

Let $\mathcal{A}_{T}$ be an attractor within the total system $S_{T}$ defined predominantly by $S_{\mathcal{M}}$ and let $x_{T}=$ $\left\{x_{E}, x_{N}, x_{M}, x_{S}, x_{\mathcal{M}}\right\}$ belongs to the set of attraction of this attractor. Then evolution of $x_{T}$ represents various processes undergoing selforganization.

Let us define the external process with respect to the main dynamical system as described by mapping $\pi_{e x t}\left(x_{T}(t)\right)=\left\{x_{E}, x_{N}, x_{M}, x_{S}\right\}(t)$ following from (1)-(5).

DEFINITION 2.1: Induction of an external state process with respect to the main dynamical system by a process of the main dynamical system $x_{\mathcal{M}}(t)$ immersed in the total state process $x_{T}(t)$ is called action of the main dynamical system.

Action is manifested by direct interaction of the main dynamical system with environment or by induction of processes in the neural network system and by this induction of action of the motor dynamical system in environment. In the second case we have to do with induction of a process in environment by $S_{M}$ and next detection of changes in this environment by the sensor dynamical system.

We expect that actions of $S_{\mathcal{M}}$ are frequent sufficiently in order to provide considerable amount of data related to properties of environment. Then, results of each action are remembered within the neural network. When some properties of environment would happen repeatedly, then our model having by assumption property of optimization of energy consuming should manifest more strong remembering of effects corresponding to these properties. By this we expect generation of relations between state of variables which are present on the input of the motor system and output of the sensor system. Consequently we suggest generation of relations reflecting properties of the environment, within neural network system. Such relations can be applied for extension of the set of formulas $F$.

Let us introduce here implication by means of the action of the main dynamical system defined above. Thus we obtain

$$
\left(x_{N M}\right)_{A} \Rightarrow\left(x_{N S}\right),
$$

where $x_{N M}, x_{N S} \in T$ and the symbol $A \Rightarrow$ stands for operation of induction of action $A$ of the system 
$S_{M}$ by means of states $\pi_{M N}^{-1}\left(x_{N M}\right)$ and appearing of the response $\pi_{S N}\left(x_{S N}\right)=x_{N S}$ from the system $S_{S}$. Let us note that action $A$ has to be considered beyond the neural network dynamical system.

Let us notice that our implication is indexed by kind of action $A$. Therefore we have to do here with a family of implications in fact. We can introduce more universal implication as

$$
\left(x_{N M} \wedge A\right) \Rightarrow\left(x_{N S}\right)
$$

In this case we have to do with two sentences on the left side of implication. The first one states that $x_{N M}$ takes a value and the second one states that action have a determined kind. Then, implication follows the sentence that $x_{N S}$ takes a determined value which can be written more formally using sentences

$$
\left(\left(x_{N M}=a\right) \wedge\left(A=A_{G}\right)\right) \Rightarrow\left(x_{N S}=b\right)
$$

which is shortly written by (8).

Let us notice that we can also consider action of $S_{\mathcal{M}}$ which happen in neural network only. To this end we can distinguish subsystems within $S_{N}$. Then, let

$$
S_{N}=\bigcup_{i}\left(S_{N M i} \cup S_{N i} \cup S_{N S i}\right) \cup S_{H},
$$

where $S_{N M i}$ is $i$-th motor subsystem within the neural network, $S_{N i}$ is $i$-th part of the neural network system, $S_{N S i}$ is $i$-th sensory subsystem within the neural network. The $S_{H}$ stands for a part of the neural network able to analyse information obtained by means of $S_{N S i}$ following from action within the system $S_{N i}$. The system $S_{H}$ also generates action in $S_{N i}$. Distinguishing of the subsystems enables us to discuss realization of action of the main dynamical system which is closed within part of the neural network.

Discussed above division of neural network into subsystems enables us to discuss implication similar to that one given by (7) which happens on the interior of the neural network only. We can consider the set of terms in relation to $i$-th subsystem by $T_{i}$. We assume as previously that they are associated with action of motor and sensor systems $S_{N M i}, S_{N S i}$ by means of the functions $\pi_{S N i}$ and $\pi_{M N i}$. They transform the set of variables of the sensory system $V_{S i}$ and motor system $V_{M i}$ into set of variables $V_{H}$ of the part $S_{H}$ of the neural network system. Thus we have $\pi_{S N i}: V_{S i} \rightarrow V_{H}$ and $\pi_{M N i}: V_{M i} \rightarrow V_{H}$.

The set of terms induced by dynamical systems $S_{N M i}, S_{N S i}$ is defined by

$$
T_{i}=\pi_{M N i}\left(V_{M i}\right) \cup \pi_{S N i}\left(V_{S i}\right) .
$$

The set $T_{i}$ contains variables associated with motor and sensor systems $S_{N M i}, S_{N S i}$ expressed within the subsystem $S_{H}$.

Consequently, the implication followed by action within the neural network system can be expressed as

$$
\left(x_{N M i} \wedge A_{N N}\right) \Rightarrow\left(x_{N S i}\right),
$$

where $A_{N N}$ represents a kind of action of $S_{H}$ in the $S_{N i}$.

Let us note that $S_{H}$ represents a system which is able to analyse results of action and transfer of input into $S_{N M i}$. However, the action is induced in fact by the main dynamical system governing the $S_{H}$.

Having at our disposal action in neural network system we have also an opportunity to comparison of the action $A$ from (9) with an action of type $A_{N N}$ created within the neural network system. Then, a model of reality represented by $A_{N N}$ is produced in case when effects of both actions are the same or similar within the neural network.

We have determined how implication appears within evolving dynamical system. By this we can imagine how proof of a theorem could happen. However, we see also that each step of the proof is 
associated with an action of dynamical system. In case when this action happens in neural network system we have the problem to what degree each step of the proof fits to reality. Each step is associated with an approximation of reality. Thereby increasing of length of the proof should lower status of formula obtained by this proof.

\subsection{Consequences of dynamical system approach for estimation of status of assumptions and theories}

Having at our disposal context provided by dynamical systems as in [1], [2] we can discuss status of assumptions in relation to formulas induced by dynamical system as well as in relation to length of proof applied in infering on form of these assumptions. We discuss also factors which decide on status of whole theory.

Let us discuss concisely some remarks obtained in [1] in order to illustrate how the term "status" can be usefull.

REMARK 2.1: Fundamental notions of theory describing reality should be related to maximum engagement by action of the dynamical system and its sensory system in order to obtain results which next can be interpreted as the simplest information by neural network system. Then, status of such a fundamental notion is viewed as high.

REMARK 2.2: Status of assumptions introduced into theory by means of proofs based on observed properties depends on length of the proof. The longer proof the lower status of assumption. This status could be increased when sevaral independent proofs starting from independent facts would lead to the same conclusion inducing just this assumption.

Above remark follows from fact that implication is considered here differently from conjunction, alternative and negation. Implication is related to action of the dynamical system what shed light on role of proof in relation to reality. The length of the proof characterizes amount of action which when increases gradually decreases correctnees of approximation of reality. It means that recognition of reality is then less direct.

Frequently we obtain representation of a function describing a physical phenomenon as expansion in a series. Then, particular terms can be associated with a physical interpretation. This can be useful for introduction of fundamental assumptions into theory. However, expansion into series depends in general on option of a basis and this option can be in fact rather arbitrary. Therefore, physical interpretation is rather risky in this case. Consequently we come to the following remark:

REMARK 2.3: Fundamental notions obtained with the aid of expansion of a function into series have low status since this expansion depends on arbitrary option of a basis. This status could be higher in case when corresponding basis would have appropriate physical interpretation. However, in general, infinite series should not have good physical interpretation since there is small chance to assign appropriate interpretation to each term. Cutting of such a series to finite number of terms is also an arbitrary option with respect to physical interpretation.

Above remark suggests that we should avoid to construct physical theory with the aid of fundamental assumptions based on expansion of a function into a series. We have also remark related to status of whole theory as follows:

REMARK 2.4: We would say that status of theory depends on number of assumptions applied to construction of this theory, status of each assumption with respect to criteria discussed previously, range of validity associated with universality of theory and with status of logic.

Above remarks are useful for constructing universal and fundamental theories of our reality by pos- 
sibility of estimation how theory fits to reality [1]. However, this methodology is not finished yet. Further ideas are expected. In this paper one introduces concept of cognition manifold which has several tasks. The first task is related to obtaining increasing of possibility of inference in relation to reality by making more clear whole methodology. The second task is related to creation of possibility of generation of context for description of reality. This context indicates how theories should evolve. Next task is associated with form of gathering of whole information on the subject how hitherto developed descriptions have been evolved. This task also supports development of the context.

Let us note that the term "status" is not entirely precisely determined. This is so since it estimates relation between internal theoretical system and external world. However we have at our disposal internal theoretical system only. By model of interactions of dynamical system with external world we can estimate what is better for recognition of reality. Consequently model of evolving dynamical system interacting with an environment allows us to understand better how the cognition process is continued. Such an understanding allows us in turn to estimate which ways of cognition are better and which are worse. This is manifested just by the term "status". Thereby we should not expect very precise definition of status. We expect rather possibility of estimation which status of assumption is higher and which is lower by inequality relation and some ordering.

We can see that obtaining of any criterion for estimation of status is success on way towards formalization of cognition process.

\section{On inductive reasoning for theoretical descriptions of reality}

Inductive reasoning is applied frequently to creation of various generalizations based on a set of experimental results. This kind of reasoning is important and frequently applied in pure theoretical physics. We would like to place this methodology within concept of cognition manifold as important method of reasoning.

Inductive thinking in relation to theoretical description is associated usually with property connected with an integer number and leads to a generalization of previous theory. Let us mention for instance inductive thinking, frequently applied in physics, on dimension of our space.

It would be convenient to have at our disposal more formal approach to this procedure as well as plecament of this procedure in methodology of whole description of reality.

Let $\phi_{I n}$ be a notion within a theory $T h_{n}$ which is associated with an integer number $n$. Then generalization of theory $T h_{n}$ to theory $T h_{n+1}$ is associated with transformation of notion $\phi_{I n}$ to the notion $\phi_{I(n+1)}$ and next development of theory $T h_{n+1}$ which should contain $\phi_{I(n+1)}$. However this development of new theory is not arbitrary.

Theory $T h_{n}$ is usually constructed taking into account a set of experimental results. Therefore the new theory also should be in accordance with this set of experimental results. We can express this fact by means of a mapping $P_{I n}: T h_{n+1} \rightarrow T h_{n}$. Mapping $P_{I n}$ should express the property that approximation of reality corresponding to processes associated with property $\phi_{I n}$ by theory $T h_{n+1}$ is sufficiently well. In other words image of mapping $P_{I n}$ considered as a theory describes reality sufficiently well in sector where $T h_{n}$ acts satisfactorily.

Consequently, we can illustrate scheme of inductive thinking placed within methodology of generating of new theories in the following general form

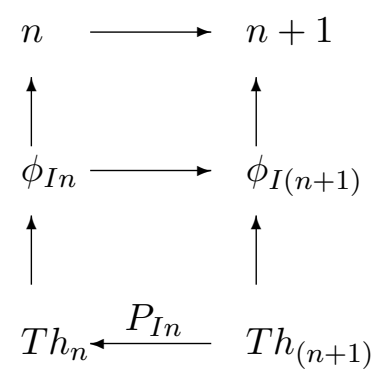


Within above scheme horizontal arrows are associated with sequential inductive steps. Vertical arrows are associated with assignation of notion undergoing induction for a given theory and also assignation of number representing step of induction for notion undergoing induction.

Let us discuss some examples of inductive reasoning. We can consider $\phi_{I n}=U_{n}\left(\mathbf{X}_{n}\right)$, where $U_{n}$ is an neighbourhood of a chosen point of space $\mathbf{X}_{n}$. Then, we can extend our theory to larger space by adding new set $\phi_{I(n+1)}=U_{n+1}\left(\mathbf{X}_{n+1}\right)$. Then our space is expressed by

$$
X=X_{n+1}=\bigcup_{i=1}^{n+1} U_{i}\left(\mathbf{X}_{i}\right)
$$

and theories describing behavior of our space have the same form. It means that the mapping $P_{\text {In }}$ represents cutting of theory from the set $X_{n+1}$ to the set $X_{n}$. This kind of induction could be named transitive since definition of the mapping $P_{I n}$ and construction of theory $T h_{(n+1)}$ is direct and evident. On this way we extend our theory into whole space which has regions not attainable for us.

Another example can be associated with scale of averaging applied in modelling. Let $\phi_{I n}=d_{n}$ be a distance which determines a volume on which properties of the medium are averaged. Then transition to next $\phi_{I(n+1)}=d_{n+1}$, where $d_{n+1}<d_{n}$ is not so direct as in previous case where theory was unchanged. Within smaller scale we see new phenomena. Then, mapping $P_{I n}: T h_{n+1} \rightarrow T h_{n}$ can be considered for instance as as $P_{I n}=D R$, where $D R$ is dimensional reduction procedure considered in [5] where multiscale modelling is discussed.

In this last case we see necessity of stopping of this inductive thinking for some $n=N$ associated with length $d_{N}$. Indeed our notion of a distance follows from possibility of recognition of this distance in our space by various phenomena including electromagnetic waves. The question is to what degree our space is three-dimensional with possibility of considering a distance in this space. In other words we have the problem how small distances we can consider at all in theoretical description.

Perhaps, for sufficiently small distances we have to activate another kind of inductive thinking. In particular this can be dimension of space. Then, $\phi_{I n}=\operatorname{dim} X$, and $P_{I n}$ will represent projection of space from larger dimension space to smaller dimension space having in mind all processes expressed in theory $T h_{n+1}$. Then, we expect that image of mapping $P_{I n}$ should approximate processes described by theory $T h_{n}$ appropriately.

Problem of stopping of inductive thinking is important. In last two cases generated theories in each step of inductive reasoning are not similar. Therefore such an induction is not direct. We should analyse which notions applied in theory have inductive character.

Let us consider notion of deformation applied in continuum mechanics. We consider there deformation function $\mathbf{x}(\mathbf{X}, t)$, where $\mathbf{X}$ is a reference configuration. When we diminish scale then we should interpret medium together with corresponding reference configuration. We obtain relative motion of something with respect to something else. Usually in smaller scale we will interpret and model this background. However, when we introduce again the deformation function for this smaller scale then we should interpret again new background for still smaller scale. Thereby, so important notion for modelling of physical processes as deformation function provokes infinite step inductive thinking. Evidently we have to pose the question how to stop such an inductive thinking. We see that some notions are open with respect to inductive reasoning.

It seems that stopping of inductive reasoning needs deep analysis of notions which are fundamental for this induction. Let us mention for instance "distance" and "direction" as fundamental for inductive diminishing of scale. We could describe theoreticaly where from such notions appear in relation to processes which are entangled in corresponding notions. Let us mention stright propagation of light or motion of single elementary particles. All they are responsible for our understanding of our geometry. Then we should organize hypotheses more fundamental than direct description of these processes and by this estimate to what degree notions associated with our induction could be changed. This in turn could provide premises for stopping this kind of induction. 


\section{Concept of cognition manifold as theoretical environment for evolution of universal and fundamental physical theories}

By means of theoretical physics we try to introduce an universal description of reality taking into account a set of observed properties or phenomena. We assume that this set should be large as far as it is possible. We try to construct models describing corresponding properties and phenomena in an unified manner. Methodology corresponding to this unified manner of description is not entirely clear and needs development. We are forced frequently to introduce various hypotheses which perhaps are not entirely verified by experimental results. Consequently, we would like to have at our disposal methods of estimation to what degree such hypotheses are appropriate in this unified description.

Methods of modelling need possibility of expression of particular observed property or phenomenon by means of theory based on a system of fundamental notions. Thereby, we should have at our disposal sufficiently complex theoretical environment for description of mentioned above properties and phenomena in order to express them correctly.

To this end, at first stage of our considerations, we introduce the set $\mathcal{B}=\left\{\left\{\tilde{X}_{p}, \lambda_{p}\right\}\right\}$ of models of our space, where $\tilde{X}_{p}$ is a geometrical space composed of points and $\lambda_{p}=\left\{\lambda_{p i}\right\}$ is a set of variables which determines fundamental states of our space considered as fields $\left\{\lambda_{p i}(\mathbf{X})\right\}, \mathbf{X} \in \tilde{X}_{p}$ on the domain $\tilde{X}_{p}$. Consequently we use here the term "space" in various context. This should be commented more precisely.

We have not introduced time yet. Our variables which determine fundamental states correspond with admissible configurations which can be attained within $\tilde{X}_{p}$. Consequently fundamental notion is connected here with the change of state of our medium. Then various variables can represent changes of various states. They can be compared. This leads to definition of time.

Time can be defined as parameter which measures relative rate for changes of one state with respect to another one. By this we can introduce processes. Consequently time is not fundamental notion. Time appears in this case as parameter related to rate of one process with respect to another which can be considered as a reference process.

Time can be rescaled in theoretical considerations. The question is whether time is changed or rate of process is changed. These problems are not equivalent. In case when we assume that time is changed then we assume in fact that all rates of processes which can be considered as reference processes for time measurement undergo the same change. This is very strong assumption which needs experimental confirmation. Fulfilling of such a condition by reality seems to be rather improbable. Therefore, it seems more reasonable to consider time classically. Change of this point of view could be based on more carefull experiments related to rate of processes.

We consider mathematical context for modelling of various phenomena which is created in our brain. Then we have at our disposal various mathematical objects. In particular we can consider a point of three-dimensional space. On the other hand we consider context related to reality which is described by a mathematical theory. In accordance with previous discussion on status of mathematical notions and theories we see necessity of considering connections beteen reality and mathematical desciption. We have stated there that theory approximates reality.

Let us note that point is important object for mathematics. However in reality point is nothing. Mathematical point is applied to approximation of reality. We observe that our vacuum has various states manifested among others by electric field for instance. Thereby our space cannot be identified with an Euclidean three-dimensional space directly since such a space has no states. Therefore real space has to be identified with a medium which is associated with a geometrical space.

Consequently our Euclidean three-dimensional space is considered as domain of fields in vacuum medium and represents an element of mathematical approximation of reality considered as a medium. Then role of single point is assigned to possibility of expression of properties of this medium within assumed method of approximation.

Frequently, in theoretical physics one considers the problem whether our space is continuous or discrete. This is not well posed question. The question is how we approximate our space. Our brain 
uses finite-dimensional neural network. Threfore we see better discrete objects by theory. Continuum description is a kind of approximation of reality. In particular we should apply continuum with finitedimensional fields for correct approximation of reality [5], [10], where scale of averaging applied to modelling is introduced in formal way.

In general methods of approximation are associated with formalization of scale of averaging applied during modelling of processes. In large scale geometry is detected by our organism by determination of direction and a distance. In smaller scale we recognize these quantities by motion of particles and also by propagation of electromagnetic waves. Therefore we consider three-dimensional space as domain for our medium.

The question is whether we can violate assumption on this dimension. This has been discussed in context of inductive thinking. We should have at our disposal hypotheses creating foundations for changing dimension of domain of vacuum medium in relation to sufficienly small scale and also mapping $P_{\text {In }}$ which joins the smaller scale models with larger scale models. Let us note that corresponding scale should be smaller than particles which determine direction by their motion. This scale should be smaller also than processes governing propagation of electromagnetic waves. However then we encounter new problem how to measure a distance. Some attempts in this direction have been done in [9].

Let us note that understanding of empty space is much more difficult than understanding of space considered as a medium. Therefore we have assumed that our space has to be considered as a medium.

At this moment of our considerations we do not introduce energy yet. Variables within $\lambda_{p}$ determine all configurations of the vacuum medium and are rather of kinematical type. Energy can be expressed by means of these variables in what follows.

Let us consider a set of phenomena observed in reality and expressed as properties $\phi_{k}, k \in I_{N}=$ $\{1,2, \ldots, N\}$. We assume that corresponding properties and phenomena are characterized by evolution of variables $\lambda_{p i}$ for a given $p$ corresponding to $p$-th model. We introduce now, as a first stage, qualitative modelling what means that we do not determine of all constants within the model but we are concentrated on fact that the model is able to describe qualitatively the chosen property $\phi_{k}$.

Let us mention for instance waving of components within vacuum medium [6], [7] or precession of electron [8] as separate phenomena for modelling. Models of these phenomena are introduced with the aid of accessible premises. It is difficult to determine immediately all properties of vacuum medium responsible for so important phenomena as evolution of wave function of electron or mechanisms of precession of electron. We try to introduce some qualitative models as first stage of description. Thereby introduced models are considered as starting point for modification of corresponding models as well as looking for opportunity for determination of constants of these models necessary in future development of whole theory of interacting phenomena.

Let us consider the phenomenon considered in reality and expressed by $\phi_{k}$. Then various models which describe this phenomenon we denote by $m_{\phi_{k} i}$. Consequently they are equivalent with respect to property $\phi_{k}$ since they describe the same property. This can be expressed by the formula

$$
m_{\phi_{k} i} \equiv_{\phi_{k}} m_{\phi_{k} j}
$$

Then whole equivalence class of corresponding models associated with the property $\phi_{k}$ is denoted by $\mathcal{M}_{\phi_{k}}$. Consequently we introduce the set

$$
\mathcal{M}_{\phi}=\bigcup_{k} \mathcal{M}_{\phi_{k}}
$$

The set $\mathcal{M}_{\phi}$ represents all models considered by theory for various phenomena observed. The question is how we should tend towards universal theory with the aid of models contained within the set $\mathcal{M}_{\phi}$.

One suggests to consider the cognition manifold as composed of the set $\mathcal{B}$ as basis on which some fibres represented by elements of $\mathcal{M}_{\phi}$ are determined. To each model we can assign its status. 
Consequently, status of elements of fibres is a function which next is useful for characterization of status of whole theory constructed.

We should have better characterization of sets $\mathcal{M}_{\phi_{k}}$ and also $\mathcal{B}$. Let us discuss structure of the set $\mathcal{B}$ in more details. Let us assume that we have several concepts of our space expressed by $\left\{\tilde{X}_{p}, \lambda_{p}\right\}$. To each $\tilde{X}_{p}$ we can apply inductive generalizations having a number of steps.

Let us introduce set of multiindexes denoted by $\tilde{I}_{L}=\mathcal{I}_{1} \times \mathcal{I}_{2} \times \ldots \times \mathcal{I}_{L}, \mathcal{I}_{l}=\left\{1, . ., N_{l}\right\}, l=1, \ldots, L$, where $l$ represents kind of induction applied, $N_{l}$ represents number of inductive step of the same $l$-th kind. Particular multiindexes are denoted by $I_{l} \in \tilde{I}_{L}$. Thereby we have then that for each $p$ we have $\left\{\left\{\tilde{X}_{p I_{l}},\left\{\lambda_{p I_{l} i}\right\}\right\}: I_{l} \in \tilde{I}_{L}\right\}$. The multiindex $\left\{p, I_{l}\right\}$ belongs to $I_{\mathcal{B}}$. Then our $\mathcal{B}$ takes the form

$$
\mathcal{B}=\bigcup_{\left\{p, I_{l}\right\} \in I_{\mathcal{B}}}\left\{\tilde{X}_{p I_{l}}, \lambda_{p I_{l}}\right\}
$$

Consequently we admit several inductive steps of one kind and next several inductive steps of another kind indexed by $l$ applied to one element of basis in order to generate next elements of this basis.

The aim of introducing of fibres $\mathcal{M}_{\phi_{k}}$ is to describe processes described in elements of $\mathcal{B}$ taking into account various ways of modelling of these processes. In particular we should obtain possibility of estimation of status of introduced notions and hypotheses in models which appear in fibres.

Our models should have connections with reality. Thereby we should have at our disposal experimental results. We assume that we have at our disposal the set of sentences $\mathcal{E}=\left\{e_{1}, \ldots, e_{m}\right\}$, which expresses just experimental results. Then, we can apply to the sentences $e_{i}$ inferring based on an assumed logic. We can create by this inferring a mathematical minimal representation of phenomena expressed with the aid of $e_{i}$. It means that we introduce the most simple formulas to description of them without any additional postulates.

Minimal mathematical representation provides a system of assumptions which have high status. They are considered as starting point for formulation of various models of corresponding phenomena with the aid of additional asumptions having lower status.

We construct fibre on basis $\mathcal{B}$ in relation to a chosen property $\phi_{k}$. The fibre is indexed among others just by $\phi_{k}$ and is composed of models which have gradually increasing number of assumptions with increasing distance from basis.

We construct first the model $m_{\phi_{k}}$ for property $\phi_{k}$ with the aid of deductive skeleton expressed by a minimal representation of $\phi_{k}$ interpreted within $\mathcal{E}$. Within this skeleton we develop more detailed models $m_{\phi_{k} i}$ by means of additional hypotheses by application of new assumptions, inductive reasoning or abduction. We try to estimate status of introduced hypotheses which should gradually become lower when we go away from basis along fibres.

On this level of constructing of theories we have to do with speculative thinking. Let us mention a remark from [1] on this kind of reasoning. Let $S t\left(A_{1}\right)<S t\left(A_{2}\right)$ means that status of assumption $A_{2}$ is higher than status of $A_{1}$. Let us consider the following remark:

REMARK 4.1: Speculative thinking needs changing of assumptions within the same theory. We should introduce hierarchy of assumptions by estimation of their status. Consequently, let $S t\left(A_{1}\right)<$ $S t\left(A_{2}\right)<\ldots<S t\left(A_{N}\right)$ be set of assumptions of a given theory ordered with respect to their status. Then, we should first change assumptions of the lowest status during speculations and gradually do it going towards the largest status assumption $A_{N}$. Consequently, $A_{N}$ should be the most stable during the speculative thinking. It means also that introduction of extra assumptions which makes constructing of theory more simple is interpreted as lack of discipline. We can admit sometimes corresponding extra assumptions. However, we should assign to them sufficiently low status.

Above remark manifests the fact that even in speculative thinking we are able to maintain a discipline and responsibility.

Let us introduce multiindex $M_{s} \in \bar{M}_{s}=\left\{\left\{\bar{M}_{s-1}, J_{s}\right\}, s \in N, \bar{M}_{1}=J_{1}, J_{s}=\{q: q \in\right.$ 
$\left.\left\{1,2, \ldots, N_{s}\right\}\right\}$. Consequently, we denote model $m_{\phi_{k}}$ by $m_{\phi_{k} M_{1}}$. It means that on the first level we can introduce $N_{1}$ models based on minimal representation corresponding to $\phi_{k}$. Perhaps the number $N_{1}$ should be equal to 1 . This is so since perhaps minimal representation should be the only one.

Within the minimal mathematical representation we introduce additional hypotheses and assumptions in order to create new models indexed by $m_{\phi_{k} M_{s}}$. We do this since our theories are usually not complete with respect to their ability to description of reality. Experimental results cannot be related to all aspects of reality. Therefore within minimal mathematical representation which has just direct relation to experimental results we carry out theoretical speculations which seem to have connections with reality with some probability. We use to this end among others intuition in order to extend context for reasoning.

As a result of this activity we obtain the relation $m_{\phi_{k} M_{s-1}}<_{A H} m_{\phi_{k} M_{s}}$. The relation $<_{A H}$ means that the model $m_{\phi_{k} M_{s}}$ is obtained directly from the model $m_{\phi_{k} M_{s-1}}$ by adding to the last model additional assumptions. Adding of new set of assumptions can be continued on various ways. Each way is represented then by element of the set $J_{s}$.

Let us explain this more precisely. Consequently, $J_{1}$ represents set of theories $\left\{T h_{i}, i \in J_{1}\right\}$ each of them is constructed with the aid of set of assumptions $T A_{i}, i \in J_{1}$. Adding new assumptions modifies theories $T h_{i}$ into $T h_{i j}$, where $j \in J_{2}$, which are based on set of assumptions $T A_{i j}, i \in J_{1}, j \in J_{2}$ and so on. We asume furtheremore that no assumptions is added when $j=1$. In other words theory $T h_{111 . .11}$ is identified with $T h_{1}$ as well as $T h_{111 . .51}$ is identified with $T h_{111 . .5}$ for instance. The last step allows us application of introduced multiindexes also for not modified theories. We identify all our models $m_{\phi_{k} M_{s}}$ with such theories assuming the same method of application of indexes.

By means of the method of indexing by multiindexes $M_{s}$ we can characterize a group of subfibres assigned to the property $\phi_{k}$. We define fibre associated with $\phi_{k}$ as set of models $\tilde{m}_{\phi_{k} n_{1} \ldots n_{s}}=$ $\left\{m_{\phi_{k} n_{1}}, \ldots, m_{\phi_{k} n_{1} \ldots n_{s}}: n_{1} \in J_{1}, \ldots, n_{s} \in J_{s}\right\}$. Then each subfibre is represented by set of multindexes $\left\{n_{1}, \ldots,\left\{n_{1}, \ldots, n_{s}\right\}\right\}$ which in turn represents models with increasing complexity. When model for a given $n_{j}$ is not modified with increasing $s$ then we endow the same model with additional index equal to 1 . Thereby some subfibres can be indexed by multiindex contaning several numbers equal to 1 . The model $m_{\phi_{k} n_{1} \ldots n_{s}}$ represents end of subfibre.

Consequently, the model $m_{\phi_{k} n_{1}}$ obtained at first stage of modelling when $s=1$ has assumptions with the highest status since they are based on minimal mathematical representation. However, this model is usually not sufficiently complex in order to satisfy our expectations in modelling. Therefore we create additional new assumptions. Next models are then obtained by additional assumptions based on hypotheses, inductive thinking or abduction. Corresponding assumptions have gradually lower and lower status with increasing of number $s$. However status of whole models as theories increases since they describe reality better owing to their larger complexity

Lack of appropriate complexity in first constructed models follows from fact that assumptions based on minimal mathematical representation indicate new ways for modelling of more complex unknown world. Let us mention an example from [9]. Observed magnetic properties of electron discussed there leads to introduction of variables which characterize state of deep structure of vacuum medium. Properties associated with corresponding variables are considered just as minimal mathematical representation. This is so since the only premises which are taken into account follow from experimental results. We need then aditional hypotheses in order to introduce more complete description.

On basis of the minimal representation we introduce in [9] deep structure of vacuum medium with states represented by magnetic field and two-ring field. Within this medium interacting magnetic monopoles can be considered. In particular network of bounded monopole-antimonopole pairs creates our space seen on more averaged level as homogeneous.

Let us assume that we have a set of properties $\phi_{k}\left(p, I_{l}\right)$. We assume that the index $k$ is related to kind of property denoted by $\phi_{k}$. Remaining indexes stand for indication of element of basis in which the property or phenomenon is expressed. We introduce our concept of cognition manifold by the following definition: 
DEFINITION 4.1: By cognition manifold we understand the following object

$$
\mathcal{M}_{C O G N}=\left\{\bigcup_{k,\left\{p, I_{l}\right\}, M_{s}} \tilde{m}_{\phi_{k}\left(\left\{p, I_{l}\right\} M_{s}\right)}, \mathcal{E}, \mathcal{S}\right\}=\{\mathcal{M}, \mathcal{E}, \mathcal{S}\}
$$

where $\mathcal{S}$ represents function which assigns status to each model.

Let us note that the function $\mathcal{S}$ assigns status to each model considered as a theory of a given phenomenon. However we have introduced previously also status of assumptions. Status of a model $m_{i}$ denoted by $\mathcal{S}\left(m_{i}\right)$ depends in general on status of assumptions $S t\left(A_{i j}\right)$ applied to formulation of this theory. Thereby we have assumed that $\mathcal{S}\left(m_{i}\right)=\Phi\left(\left\{S t\left(A_{i j}\right)\right\}\right)$, where $\left\{A_{i j}\right\}$ is set of assumptions for $i$-th model.

Let us assume that we have estimated status of each model describing a given phenomenon $\phi_{k}\left(\left\{p, I_{l}\right\}\right)$ as $S\left(m_{\phi_{k}\left(\left\{p, I_{l}\right\} M_{s}\right)}\right)$. We define cross-section within the set $\mathcal{M}_{C O G N}$ as follows

$$
\mathcal{C}\left(\mathcal{M}_{C O G N}\right)=\left\{\bar{m}_{\phi_{k}\left(\left\{p, I_{l}\right\}\right)}: \bar{m}_{\phi_{k}\left(\left\{p, I_{l}\right\}\right)} \leftarrow \max _{M_{s}} S\left(m_{\phi_{k}\left(\left\{p, I_{l}\right\} M_{s}\right)}\right),\left\{p, I_{l}\right\} \in I_{\mathcal{B}}\right\},
$$

where $\leftarrow$ means operation of option of the model having the highest status within set of models placed at the end of each subfibre. The end of subfibre means that $s$ stands for largest number attained during modelling. By taking the cross-section we chose one element within whole group of subfibres indexed by $M_{s}$ and related to a given fibre indexed by $\phi_{k}$. The option is based on estimation of status of the model and its comparison with status of other models within group of last elements of subfibres indexed by $M_{s}$ and defined by application of the relation $<_{A H}$. In other words we assign one, the best model of a given phenomenon $\phi_{k}$ to a given fibre within our way of speculation. We do it for each phenomenon considered during description.

The set $\mathcal{C}\left(\mathcal{M}_{C O G N}\right)$ can be decomposed into the sets

$$
\mathcal{C}\left(\mathcal{M}_{C O G N}\right)=\bigcup_{\left\{p, I_{l}\right\}} \mathcal{C}_{\left\{p, I_{l}\right\}}=\bigcup_{\left\{p, I_{l}\right\}}\left\{\bar{m}_{\phi_{k}\left(\left\{p, I_{l}\right\}\right)}\right\}
$$

The set $\mathcal{C}_{\left\{p, I_{l}\right\}}$ represents models which together describe all chosen properties of reality acssociated with element of basis $\mathcal{B}$ indexed by multiindex $\left\{p, I_{l}\right\}$. However, in order to construct model of all considered phenomena which can interact, we should join selected models. Consequently, by theory of reality composed of all models within $\mathcal{C}_{\left\{p, I_{l}\right\}}$ we understand

$$
\mathcal{C}_{J\left\{p, I_{l}\right\}}=\left\{\mathcal{C}_{\left\{p, I_{l}\right\}}, \mathcal{J}_{P R O C}\right\}
$$

where $\mathcal{J}_{P R O C}$ means procedure of joining of models from $\mathcal{C}_{\left\{p, I_{l}\right\}}$. This procedure can be associated for instance with division of space represented by vacuum medium determined in basis $\mathcal{B}$ into parts $V=\bigcup_{m} V_{m}$. Then joining of models can be realized by means of boundary conditions determined on $\partial V_{m}$. The procedure can be also associated with introduction of additional postulates for variables of joined models related to energy for instance and various forms of constitutive equations.

Our method of modelling by theoretical speculations rests on modelling of separate phenomena on speculative way and joining of corresponding models into one more universal theory. However we do our speculations on one plane determined by basis element of cognition manifold. Procedure of defining cross-section within cognition manifold is not simple. We have to take into account status of speculative theories. This should be done considering many sources for estimation of this status. Let us mention accordance with experimental results, status of mathematical theories applied, including status of logic, and methodology of introducing of assumptions. We should admit also the case when methods of joining of models corresponding to one element of basis can have impact on option of form of cross-section. Several comments on this subject will be done in what follows.

Summarizing we have introduced concept of cognition manifold which provides environment for introduction of new theories, their evolution and estimation of their relations with reality. We have to do here with set of theories indexed by elements of basis $\mathcal{B}$. The most universal and fundamental theory have to be selected just from this set. 


\section{Cognition manifold as manifestation of qualitative reasoning towards recognition of reality}

By introduction of cognition manifold we accentuate role of qualitative reasoning. We do it by construction of qualitative models and corresponding equivalence classes for models describing the same phenomenon. The question is what for such a qualitative approach to description of reality is introduced.

In order to catch large set of phenomena within one theory we develop a strategy. This strategy rests on constructing a network of fundamental notions and models which would be large sufficiently in order to describe all phenomena. In this place we see tendency to construction of universal theory.

Evidently we are not able to carry out all experimental results in relation to whole reality. Therefore role of hypotheses is important on our way. On the other hand we cannot develop too precise models of particular phenomena since flexibility of modelling corresponding to larger context will be smaller.

Thereby having sufficiently large network of qualitative models we continue our strategy by going to next step which consists in shrinking of our network. We do it by various cross-cections within cognition manifold. Then we obtain more consistent and universal theories. When we are sufficiently satisfied having in mind consistency of our qualitative theory we go to the next step. This step rests on identification of constants in order to obtain fundamental and universal quantitative theory.

We would like to accentuate fact that reasoning based on qualitative properties can be entirely precise from formal point of view. This reasoning is based on appropriate definition of corresponding equivalence classes. These classes need not contain finished models. However, we should have evidence that corresponding models are realizable. Then, we have not to consider too large number of details within initial formulation of our theories. This in turn gives us opportunity for manipulation of larger network of theoretical models for recognition of reality.

When we try to describe reality in an unified manner we encounter obstacles. One of them is associated with very complex mathematics which represents precise reasoning with large number of details. The second one is related to option which way of description is appropriate having in mind considerable large number of possible descriptions and necessity of doing speculative hypotheses.

Above obstacles have opposite character. On the one hand we need precission in details. On the other hand we would like to find optimal way in this environment by manipulation of introduced descriptions. Therefore we are forced to find a compromise. Qualitative reasoning seems to be just such a compromise. It provides us considerable flexibility in modelling. When we obtain satisfactory qualitative description we can continue development of our description. We go then towards taking into account larger number of details within chosen representatives of corresponding equivalence classes representing qualitative properties. Finally we should obtain on this way precise mathematical description of whole reality.

Let us note that we frequently encounter in theoretical physics comments which suggest that qualitative reasoning is a preliminary stage in construction of physical theory. This in turn means that quantitative theory is more important as directly confirmed by experimental investigations and is seen then as serious achievement. Perhaps we should change this point of view.

Quantitative theory can be successful in some range of validity. However some phenomena do not exist within such a theory. It means that this theory has loss of quality in this region of description. In other words this theory is not universal. The question is what is more important for theoretical physics qualitative theory which describes all phenomena observed or quantitative theory which describes a part of phenomena only. Within this work one suggests that qualitative universal theory is more important against currently dominant point of view that quantitative theories are more important. This is so since qualitative universal theory is appropriate stage in development of theoretical physics leading to indeed universal theory.

Consequently qualitative reasoning can lead to preliminary stage of theoretical description. However such a reasoning can also be associated with very complex and by this advanced qualitative theory expressed within cognition manifold for instance. 
Above discussion shows that we develop theoretical physics with the aid of mathematics. However larger efforts are done within this methodology in order to introduce flexibility in making asumptions. Role of this flexibility will be seen especially in next sections where proof with respect to reality is discussed. This situation differs from traditional mathematical physics where we consider rather stable set of assumptions. Then, in mathematical physics, we are interested in precise formulation of theory and formulation of various theorems precisely proved.

REMARK 5.1: Formalization of cognition process changes methodological situation for mathematical physics. One shifts accent from precise mathematical reasoning by theorems towards introduction of more flexible systems of assumptions leading in fact to larger number of theories. Then qualitative reasoning allows us to discuss simultaneously this larger number of theories and manipulate them in order to fit description to reality better. However finally, after stage of specualtions, we will be interested in precise mathematical formulations of various theorems within the best theory selected.

\section{An example of constructing of cognition manifold}

\subsection{Elements of cognition manifold characterized for vacuum medium related to scale $S_{V M}$}

We discuss here a general framework for speculations within cognition manifold leading to vacuum medium mechanics. Within this section we try to show how process of modelling based on speculations is initiated. Considerations carried out here are related to scale of averaging $S_{V M}$ smaller then size of stable elementary particles. Therefore such particles are considered as extended particles and are manifested by processes within vacuum medium. Then components of corresponding particles are represented by their densities.

In order to characterize methods of speculations related to vacuum medium carried out within cognition manifold we should introduce elements of basis of cognition manifold as first step.

Various cosmological investigations indicate that the space of the Universe is flat. Furthermore, investigations of background radiation show existence of a resting reference frame with respect to radiation in our space. In particular we are able to determine velocity of Earth with respect to this reference frame. Above observations suggests that vacuum medium is similar to solids. Just such a medium is able to maintain the reference configuration and corresponding reference frame. In case when vacuum medium would be similar to a fluid we could expect varying physical laws going from one point of space to another.

Our space is rather not empty. We can detect existence of electric field for instance what represents a state of our space. This means that we have to consider our space as a medium associated with geometrical space. Our physical laws seem to be the same in all points of space or in all neighbourhoods of corresponding points.

Creation of electron positron pair suggests that this process is associated with separation of some components. Possibility of annihilation of these particles supports this point of view since corresponding components can also be joined again. Consequently we should assume that our space is identified with a vacuum medium similar to solids, composed of various components which could be separated when higher energy is provided to this medium.

Having in mind above remarks we consider our vacuum medium as similar to solids for low energy with distinguished reference configuration corresponding to three-dimensional space $E^{3}$, where we can introduce a Cartesian coordinate system.

The vacuum medium is considered as a mixture of four components [15] joined within an elementary unit identified at this moment with a point of space. Consequently elementary units have stable positions within our model of vacuum medium what reflects fact that we have intention to consider 
the vacuum medium as similar to solids for low energy state.

Motivations for assuming four components follows from observation that the Maxwell equations exhibit a symmetry with respect to electric and magnetic field. This induces considering at least two components. However, creation of electron-positron pair indicates that a separation of components is associated with electric field only. Therefore, two components are assigned to electric field. By analogy to observed symmetry between electric and magnetic fields two other components are also assigned to the magnetic field.

We have assumed here that these components constitute four-component elementary units which create a stable medium for low energy states. In the continuum description applied here the elementary units correspond to points of space. However, in particular cases we can also assume that elementary units have a finite size which is more natural and convenient in our considerations.

We introduce densities which represent an amount of component related to a volume which can be discussed owing to the introduced coordinate system. Thus, we assume that $\varrho_{v}, \varrho_{\bar{v}}, \varrho_{w}, \varrho_{\bar{w}}$ and $\varrho$ stand for densities of the components and the density of the united media, respectively. We have then

$$
\varrho_{v}+\varrho_{\bar{v}}+\varrho_{w}+\varrho_{\bar{w}}=\varrho .
$$

State of each elementary unit is described by displacements or a kind of polarization of discussed components within units. They are represented by vectors $\mathbf{v}, \overline{\mathbf{v}}, \mathbf{w}, \overline{\mathbf{w}}$. We assume that two pairs of the components are discriminated by special interactions. Components within each pair are able to move with respect to each other. As a result, we can reduce in some cases the number of variables by introducing the new ones: $\mathbf{u}=\mathbf{v}-\overline{\mathbf{v}}$ and $\mathbf{q}=\mathbf{w}-\overline{\mathbf{w}}$. At this moment it is also assumed that $\overline{\mathbf{v}}=-\mathbf{v}, \quad \overline{\mathbf{w}}=-\mathbf{w}$.

The variables $\mathbf{u}$ and $\mathbf{q}$ are identified with the vector of the electric field intensity $\mathbf{E}$ and the magnetic induction vector $\mathbf{B}$, respectively. Propagation of $\mathbf{u}$ and $\mathbf{q}$ through the vacuum medium is interpreted as electromagnetic wave.

In the paper [15] we have assumed that the medium $\varrho$ can be decomposed into the sum $\varrho=a+b$ for higher energy, where

$$
a=\varrho_{v}+\frac{1}{2}\left(\varrho_{w}+\varrho_{\bar{w}}\right)
$$

and

$$
b=\varrho_{\bar{v}}+\frac{1}{2}\left(\varrho_{w}+\varrho_{\bar{w}}\right) .
$$

This decomposition appears as a result of attaining by $\mathbf{u}$ a critical value $\mathbf{u}^{*}$ characteristic for the discussed medium.

These relations have symbolic character which accentuates that an internal structure is associated with such a decomposition process. Symbolic character means that we are not able to interpret this decomposion entirely. Some efforts on this way are done in next subsection where we discuss approach to modelling related to still smaller scale.

Components $a$ and $b$ create medium of electron and positron respectively when they rotate. In such a case the components are separated from the remaining part of the medium by a discontinuity surface. Then, motion of this surface determines of motion of electron or positron.

Deformation of the whole $\varrho$-medium is also considered. We describe this deformation by means of variable $\mathbf{h}$.

We also admit rotation of a part of the $\varrho$-medium separated by a discontinuity surface from remaining part of stable $\varrho$-medium. Such a state is identified with neutrino and motion of the discontinuity surface describes motion of the neutrino.

We have discussed concisely some assumptions related to vacuum medium what allows us to characterize states of this medium. Consequently we are able to characterize an element of basis of cognition manifold $\mathcal{B}=\left\{\mathcal{B}_{p}\right\}=\left\{\left\{\tilde{X}_{p}, \lambda_{p}\right\}\right\}$. 
We can consider several elements of basis at this stage of considerations. Option of variant will depend on further steps of development of description of processes.

Let us introduce first element of basis $\mathcal{B}_{1}$. We have introduced four-component vacuum medium similar to solids with geometry expressed by $\tilde{X}_{1}=E^{3}$ and states $\lambda_{1}=\{a, b, \varrho, \mathbf{u}, \mathbf{q}\}$.

When we take into account gravitation then we should consider various additional variants. They can be associated with existence of fifth component of vacuum medium or additional state of fourcomponent vacuum medium. Then $\mathcal{B}_{2}$ can be extended from $\mathcal{B}_{1}$ by adding new component and also by adding new state corresponding to displacement $\mathbf{h}$. We can also consider new state corresponding to displacement $\mathbf{h}$ without assumption on existence of aditional component. Then $\mathbf{h}$ is interpreted within existing components what leads to element $\mathcal{B}_{3}$ of basis of cognition manifold.

We see that various elements of basis can be determined at the same scale of averaging related to description of vacuum medium. More precise option of elements of basis should be carried out during modelling of processes.

We have introduced several elements of basis of cognition manifold. Next step consists in determination of fibres. They are based on division of space of states of vacuum medium into sectors. Let us discuss concisely various processes in vacuum medium.

We assume that each component $\varrho_{v}, \varrho_{\bar{v}}, \varrho_{w}, \varrho_{\bar{w}}$ considered separately attracts its own elements. Components $a$ and $b$ have the same property. Attraction between various kinds of components takes place for sufficiently small energy what leads to formation of the elementary units. Components $a$ and $b$ after separation also exhibit attraction which can lead, for some conditions, to recovering of the elementary units and thereby the four-component vacuum medium structure.

We could consider several energetic levels with different kinematics. The lowest energy is connected with displacements $\mathbf{u}$ and $\mathbf{q}$ only, which are considered as small. Higher energy levels are associated with elementary particles. Consequently, electron and positron are viewed as rotating $a$ and $b$-media separated from $\varrho$ by a discontinuity surface. Interactions between particles and electromagnetic field are determined with the help of boundary conditions given on the discontinuity surface.

One also admits coexistence of components $a, b$ and $\varrho$ in the same point of space. Such a state is considered as a nonequilibrium state of the vacuum medium and has to relax to a lower energy level. In particular when electron moves then motion of the separation surface is associated with production of an excess of $b$ before the particle and transfering of $a$ into the particle as well as stopping part of $a$ behind the electron. This induces just nonequilibrium distribution of components around the particle. Consequently $\{a, b, \varrho\}(\mathbf{X})$ associated with moving particle is interpreted as the wave function.

Fibres of cognition manifold contain models related to distinguished processes denoted by $\phi$. Let us note that we use the term processes. It means that time is introduced into considerations. This is done in way discussed in section where the cognition manifold is introduced and time is defined. Thereby within fibres we use all notions admissible for creation of theory since fibres are composed of models. In particular energy conservation law can be formulated and introduced in context of states corresponding to basis and defined next processes.

Let $\phi_{1}$ be related to low energy processes without any separation of components. Then states are considered as $\{\mathbf{u}, \mathbf{q}\}$ and lead to description of electromagnetic waves and static fields.

When we admit separation of components then $\phi_{2}$ can be associated with dynamics of separated component $a$ for instance. However dynamics can be associated with relaxation of this excessive amount of component or with integration of it into electron. Then $\phi_{2}$ could be divided and replaced by $\phi_{2}$ and $\phi_{3}$. Then $\phi_{3}$ represents states corresponding to internal dynamics of components within electron.

Let us mention that stable vacuum medium can be forced into rotation. Such a process can lead to creation of neutrino. Then $\phi_{4}$ can be associated with state of vacuum medium corresponding to relative displacements of one part of $\varrho$-medium with respect to another and dynamics of a slip on discontinuity surface. Let us mention that we have to do here with additional variables corresponding to displacemens. This suggests in turn that we have to do with next element of basis of cognition manifold. 
We have shown above methods of option of fibres. Development of fibres is associated with creation of additional assumptions and models corresponding to various $\phi_{k}$.

Whole theory of vacuum medium should consider a set of $\phi_{k}$ which represents all admissible states and processes within this medium. Let us note that assumptions related to form of energy conservation law for instance appear just during constructing fibres. In this place various forms of consitutive equations can be considered.

Let us discuss several examples from literature which can be represented by various $\phi_{k}$. In [18] we consider behavior of static electric field in vicinity of electron considered as extended particle. One shows that such a field should be flat in some region and should take form of Coulomb field at larger distances from electron. In [8] one consideres model of precssion of electron with dominant mechanisms called there surface superfluidity property expressed by properties of the vacuum medium. Waving associated with nonequilibrium distribution of components within stable vacuum medium is discussed in [6]. Models of neutrina and their interactions are discussed in [17]. Processes on the inside of black holes are discussed in [16].

All above models discussed in literature and considered separately can be seen as interesting concepts having perhaps not too much with reality common. In traditional approach physicists should expect necessity of experimental confirmation of such hypotheses. However when we consider all above examples within methodology manifested by the cognition manifold then all they appear as consistent action towards recognition of reality. In case when we introduce estimation of stataus of assumptions and theories then such speculations are joined with a reality not necessarily by experimental observations. Furtheremore such speculations can lead to seeing of entirely new reality within well known set of experimental results which is at disposal of physics.

Let us mention surface superfluidity property [8] as property of vacuum medium responsible for precession of electron. We assume that volume of component $a$ in electron is considerably smaller than the same amount of $a$ in $\varrho$. This is property with high status. Then interactions between $a$ and $a$ from $\varrho$ have nonhomogeneous character owing to transition of attractive interactions through the discontinuity surface. Then we can expect dynamics leading to stationarization of corresponding interactions generating tangent to the surface forces. This in turn should be manifested by evolution of motion of $a$ towards a constant slip velocity on border between $a$ and $\varrho$. Status of such a point of view is relatively high owing to direct reasoning. This means that concept of surface fluidity property is justified even in case when we are not able to carry out experimental investigations.

Interpretations leading to description of entirely new reality happen just in development of vacuum medium mechanics. Within existing set of experimental results vacuum medium mechanics sees that proton is composed of three positrons [19], [9]. Neutrina can create unstable bounded states with charged particles [17]. All this leads to entirely new classification of elementary particles [9]. There are no quarks. Boson $\mathrm{Z}$ is not carrier of electroweak interactions in direct interactions between electron and neutrino. Big Bang model is considered as explosion of space on the inside of giant black hole [16] and leads to processes which are in accordance with astrophysical observations.

Estimation of status of various assumptions applied will be considered in discussion related to proofs with respect of reality in what follows.

\subsection{Elements of cognition manifold characterized for vacuum medium related to scale $S_{D V M}<S_{V M}$}

Various considerations related to dynamics of processes, especially related to biology, suggest that dynamics of wave function is of key importance for selforganization process [11], [10], [7]. Thereby dynamics of this function can be associated with driven force for biological evolution. Within vacuum medium model related to scale $S_{V M}$ wave function is represented by $\{a, b, \varrho\}(\mathbf{X})$. Thereby understanding of physics of components in more detail seems to be important task.

In order to do it we should carry out inductive step to scale $S_{D V M}<S_{V M}$. Then we can have a hope to see structure of the wave function. We should look for premises for modelling of processes in so small scale. 
Let us note that electron is considered as electric monopole since it generates electric field perpendicular to its surface. On the other hand we do not observe magnetic monopoles. However electron has magnetic properties. The question is where from such properties follow. We can introduce concept that magnetic monopoles are on the inside of electron and therefore they are responsible for its magnetic properties. However then electron should generate magnetic field in perpendicular to its surface direction. Such a point of view suggests that an anisotropy in vacuum medium could happen. Such anisotropy should be caused by states of vacuum medium related to additional components in comparison with introduced previously magnetic ones in structure of vacuum medium. Consequently we have to do here with reasoning related to smaller scale than $S_{V M}$ since we try to see elements of vacuum medium within component $a$ and also new components related to smaller scale and responsible for mentioned anisotropy.

By means of above reasoning we obtain a premise for assumption that perhaps electron is composed of magnetic monopoles as main elements of component $a$. Positron could be composed of antimonopoles as main elements of component $b$. Then stable vacuum medium should be composed of network of systems composed of monopole and antimonopole in bounded state. Consequently network of such systems has structure similar to solids. However we have to introduce description which ensures that propagation of external magnetic field from monopole is not continued in perpendicular to its surface direction. This is necessary since electron has not magnetic field with components perpendicular to its surface direction.

Mnopole-antimonopole system moving approximately on a sphere without any polarization would be considered as an atom of $\varrho$-medium. Polarization of their motion on the sphere would lead to electric field. The polarization can be manifested by motion of monopoles around poles of sphere determined by an axis represented direction of electric field. Such an interpretation of electric field is justified by fact that electric field is associated with separation of components. Consequently, sufficiently strong polarization of monopole-antimonopole system leads to separation of them and providing monopole to electron in case of its motion. Thereby within deep structure of vacuum medium related to scale $S_{D V M}$ electric field is not defined between monopoles.

Various concepts of state of deep structure of vacuum medium leading to creation of monopole are admissible. Let us assume that our monopole is produced as a result of separation of components $\varrho_{\mathbf{w}}$ and $\varrho_{\overline{\mathbf{w}}}$ owing to incresing of magnetic field $\mathbf{w}$ and $\overline{\mathbf{w}}$ from

$$
\varrho_{\mathbf{q}}=\varrho_{\mathbf{w}}+\varrho_{\overline{\mathbf{w}}}
$$

considered as a part of the whole vacuum medium representing the background neglecting at this moment additional components. Background vacuum medium is understood here as vacuum medium without any monopoles.

We assume that the monopole is created after separation of its component and undergoes rotation taking shape similar to a sphere. This happens owing to nonlocal attracting forces within each component of the medium and forces which act on the surface of particle. This happens in similar way as for electron but forces considered here can have another character.

Consequently, we consider a monopole composed of $\varrho_{\mathbf{w}}$ separated from $\varrho_{\mathbf{q}}$ by a discontinuity surface $\mathcal{S}_{\mathrm{w}}$. Then, motion of this surface corresponds to motion of the monopole.

The surface $\mathcal{S}_{\mathrm{w}}$ is considered as a discontinuity surface for various fields describing state of the medium. In particular densities of components are changing on $\mathcal{S}_{\mathbf{w}}$ in a discontinuous way. Thereby, in order to analyze motion of our particle we should consider various balance equations taking into account discontinuity surface within assumed continuum description.

In order to prevent efficient propagation of magnetic field in perpendicular direction to surface of monopole we assume that additional components take part in near-to-surface of monopole processes. Such processes govern motion of surfaces of monopoles and propagation of magnetic field. Manifestation of the additional components is realized by the two-ring field.

We have discussed above some aspects of existence of magnetic components only. However we have postulated existence of components responsible for anisotropy of magnetic field near monopole. Let us 
denote them by $c_{\mathrm{w}}$ and $c_{\overline{\mathrm{w}}}$. Two additional components are introduced since we accept symmetry related to behavior of monopoles and antimonopoles. We should characterize states of these components which should lead to discussed previously anisotropy.

Let us introduce the vector $\mathbf{n}_{\eta}$ which can be interpreted as normal to surface of monopole. This vector determines plane tangent to surface of monopole. On this surface the two rings appear. On the other hand we consider each ring of the two-ring field as having radius denoted by $\eta_{e}$ and $\eta_{i}$ correspondingly. We introduce the variable $\eta$ as follows

$$
\eta \mathbf{n}_{\eta}=\left(\eta_{e}-\eta_{i}\right) \mathbf{n}_{\eta} .
$$

Motivation for introduction of the two ring field are as folows: (a) the fact that the variable $\eta$ should have opposite sign on surfaces of opposite monopoles what leads to two rings and (b) the fact that propagation of magnetic field in tangent to the surface of monopole direction should differ from propagation in normal to the surface direction which discriminates tangent plane. Thereby variables $\eta_{e}$ and $\eta_{i}$ are interpreted as states related to components $c_{\mathrm{w}}$ and $c_{\overline{\mathbf{w}}}$ correspondingly.

The property (a) is introduced in order to obtain possibility of modelling of explosion of space after creation of bounded state of monopoles based on repulsion following from properties of the two-ring field. Then after separation of components volume of dense component $a$ in electron is considerably smaller than volume of network of bounded monopole and antimonopole systems with the same amount of $a$. Then component $a$ within electron is considered as dense fluid composed of the same type of monopoles. For more detailed discussion see [9].

Let us note that variables in (26) are introduced as a minimal mathematical representation of properties (a) and (b). It means that existence of corresponding fields has relatively high status. This is so since properties (a) and (b) are closely related to observed properties and minimal mathematical representation, in similar way as in Remark 2.1, introduces the simplest relation without any additional assumptions. Consequently variables introduced by (26) are apropriate starting point for further speculations.

Having at our disposal description of states of vacuum medium related to scale $S_{D V M}$ we can characterize an element of basis of cognition manifold $\mathcal{B}_{D V M}=\left\{\mathcal{B}_{D V M p}\right\}=\left\{\left\{\tilde{X}_{D V M p}, \lambda_{D V M p}\right\}\right\}$.

We have that element $\mathcal{B}_{D V M 1}$ is characterized by $\tilde{X}_{1}=E^{3}$ as medium corresponding to space between monopoles. States of such a medium can be represented by $\lambda_{D V M 1}=\{\mathbf{w}, \overline{\mathbf{w}}, \eta\}$.

Element $\mathcal{B}_{D V M 1}$ is seen as the simples one. It seems that $\mathcal{B}_{D V M 2}$ should be related to $\lambda_{D V M 2}=$ $\left\{\mathbf{w}, \overline{\mathbf{w}}, \eta_{e}, \eta_{i}, \xi\right\}$, where $\xi$ represents groups of variables which characterizes state of components $c_{\mathbf{w}}$ and $c_{\overline{\mathbf{w}}}$.

Let us note that we have to distinguish four-component deep structur of vacuum medium. This is obtained by means of premises elaborated and expressed within minimal mathematical representation from (26). One suggests furtheremore that components $c_{\mathbf{w}}$ and $c_{\overline{\mathbf{w}}}$ are responsible for solids like structure of vacuum medium in small scale to largest degree. Thereby our speculations subordinated to a discipline following from cognition manifold methodology lead in fact to a discovery. This discovery has rather low status however allows us to continue cognition process.

On the other hand we encounter necessity of stopping of inductive reasoning. In case when monopoles appear as a result of separation of components from $\varrho_{\mathbf{q}}$ and corresponding rotation we have to do with relative motion and perhaps necessity of considering new background vacuum medium related to smaller scale than $S_{D V M}$.

In order to stop this process of inductive decreasing of scale perhaps we could admit the case when four components $\left\{\varrho_{\mathbf{w}}, \varrho_{\overline{\mathbf{w}}} c_{\mathbf{w}}, c_{\overline{\mathbf{w}}}\right\}$ create solids like structure with various polarizations or small displacements. Then within such a medium we could admit structural phase transformations leading to various forms of reorganization of components and interactions between them. In this place we see role of fibres which need determination of energy for description of structural transformations perhaps admitted in various variants.

Consequently, monopole would correspond with state obtained after exceeding of a critical value for magnetic field. Furtheremore transition to monopole would be associated with transformations 
in components $\left\{c_{\mathrm{w}}, c_{\overline{\mathrm{w}}}\right\}$ leading to discussed previously anisotropy in neighbourhood of surface of monopole. In this case we avoid separation of magnetic components and its motion in a background. Thereby inductive reasoning is to some degree stopped since we do not consider new background medium.

All this provides premises for speculations on form of elements of basis of cognition manifold related to scale $S_{D V M}$ and development of new models. Let us note that owing to introduction of iductive step towards smaller scale we have also imposed limitations on freedom in speculations by mapping $P_{I n}$. By means of this mapping transition to scale $S_{V M}$ is continued. It means that properties observed for scale $S_{V M}$ have to be expressible in scale $S_{D V M}$. Let us mention for instance modelling of electromagnetic waves for scale $S_{D V M}$ and verification of such a model for scale $S_{V M}$ after averaging.

Let us notice that relations (23), (24) can be reinterpreted. Components $\varrho_{v}, \varrho_{\bar{v}}$ represent in fact magnetic monopoles in averaged way and therefore they are related to scale $S_{V M}$. Components $\varrho_{w}$, $\varrho_{\bar{w}}$ are related to background vacuum medium and by this to scale $S_{D V M}$. However they can be formally expressed also directly in scale $S_{V M}$. This explains why Maxwell equations are not entirely symmetrical with respect to electric and magnetic field.

\section{Selforganizing inference as important for recognition of physical reality towards better theory}

Concept of cognition manifold expresses fact that we are frequently present in theoretical environment generated by various hypotheses not necessarily well associated with direct experiment. Furtheremore we should tend towards improvement of models by increasing of their status. We are accustomed to carry out precise mathematical proofs and consider obtained consequences as appropriate for description of reality. This follows from our traditional interpretations of mathematics as precise tool for reasoning. However, physical reality is approximated by precise mathematical theory only. In order to reflect this fact status of theory as well as various assumptions are introduced. The question is how we should follow going towards increasing of status of theory.

It seems that construction of more appropriate theory, having higher status, should be preluded by various theories of lower status. Theories of low status are then associated with hypotheses and their verification provides extension of context for determination of better theory. We would say that evolution towards theory better fitting to reality $T h_{F}$ is seen as a selforganization process

$$
T h_{1} \rightarrow_{\mathcal{C}_{1}} T h_{2} \rightarrow_{\mathcal{C}_{2}} \ldots \rightarrow_{\mathcal{C}_{F-1}} T h_{F} \equiv T h_{R},
$$

where $T h_{1}$ is theory considered as starting point for this process. This process should be sufficiently long in order to obtain a theory $T h_{R}$ as the most close to reality.

The question is what circumstances induce transition $T h_{k} \rightarrow \mathcal{C}_{k} T h_{k+1}$. The answer to this question discussed in [1] states that each step of transition to better theory is associated with context $\mathcal{C}_{k}$ which provides premises for such a step.

Let us introduce mapping $I_{E X}: \mathcal{E} \rightarrow \mathcal{M}$ which assign interpretation of the sentence $e \in \mathcal{E}$ within models contained in $\mathcal{M}$. By interpretation we understand description of process within a given model which leads to result expressed by the sentence $e$. Consequently, we obtain the possibility of estimation of accordance of corresponding model with experimental observations.

We can consider the situation when $I_{E X}(\mathcal{E}) \subset \mathcal{M}$ and $D_{E X}=\mathcal{M}-I_{E X}(\mathcal{E}) \neq \emptyset$. It means that some models have no connection with obtained set of experimental observations.

We can identify our theory $T h_{1}$ with an $\mathcal{C}_{J\left\{p, I_{l}\right\}}$. Then, we can identify all constants of the theory by means of experimental results in case when $\mathcal{C}_{J\left\{p, I_{l}\right\}} \subset I_{E X}(\mathcal{E})$. Consequently, we would say that we can estimate status of our theory by direct identification path associated with mapping $I_{E X}$. Then development of context consists in formulation of new hypotheses which would be able to improve accordance with experimental results on qualitative and quantitative way. 
However, we can also have to do with the case when $\mathcal{C}_{J\left\{p, I_{l}\right\}} \subset D_{E X}$. This can be the case when our models are related to very small scale when direct experiments cannot be carried out. However, then we can go along identification path associated with mappings $P_{I_{k}}$ introduced in (13) towards regions belonging to the set $I_{E X}(\mathcal{E})$. Then in order to make this procedure more systematic the mapping $P_{I_{k}}$ should be extented to mapping $\tilde{P}_{I_{k}}$. The mapping $P_{I_{k}}$ realizes transition of a one model into larger scale model for instance, where experimental results can be expressed. The mapping $\tilde{P}_{I_{k}}$ should realize transition form all models obtained in speculatve way into model where experimental results can be attained. In other words $\tilde{P}_{I_{k}}$ represents a family of mappings of $P_{I_{k}}$ type.

Having at our disposal such mappings we can discuss incompatibility with experimental results since they can be tranferred by means of mappings $\tilde{P}_{I_{k}}^{-1}$ into appropriate level of modelling creating just context $\mathcal{C}_{1}$ for our theory $T h_{1}$. By this step we can analyse character of incompatibilities and possible methods of improvement of this situation. Let us note that the mapping $\tilde{P}_{I_{k}}$ is usually not one-to-one. Therefore $\tilde{P}_{I_{k}}^{-1}$ can create new space for theoretical speculations.

We see in this place important role of mapping $P_{I_{k}}$. It is important to construct whole cognition manifold in way where each element of basis $\mathcal{B}$ can be connected by corresponding mapping with element where current experimental results can be interpreted.

We can summarize this situation as follows. The mapping $I_{E X}(\mathcal{E})$ provides a projection of experimental results on cognition manifold. Then, theories which are placed on the outside of this projection can be joined with experimental results by an identification path associated with appropriate mappings within the cognition manifold. Then, confrontation of theory with experimental results by means of these mappings extends context of this theory.

Let us comment role of context in this case. We consider various aspects of fitting of theory to experimental results. We can analyse direct deviation of theoretical results from possible large spectrum of experimental results. However we can discuss also quality of accordance betwen theory and experiment. In other words we discuss whether all observed phenomena are represented within theory. Then context is associated with all points of view on questions where from deviations could follow or why some effects are present within theory or not. This in turn can be starting point for creation of new hypotheses.

The context $\mathcal{C}_{1}$ can be developed also by all possible ways and additional concepts relating to more general points of views. Corresponding discussion can lead to reformulation of hypotheses which would have at this moment higher status since they take into account additional information. All this leads to determination of new theory $T h_{2}$ which is starting point for development of the context $\mathcal{C}_{2}$.

We can also try to extend the set $\mathcal{E}$ by extension of experimental investigations. However this task becomes more and more difficult for more fine processes where experimental investigations are more strongly conjugated with theoretical investigations. This elucidates role of mapping $I_{E X}(\mathcal{E})$ which join experimental results with currently developed models. Thereby interpretation of experimental results depends on models introduced. This in turn can suggests new kinds of experiments.

Summarizing we see that selforganizing inference on properties of reality, manifested by starting point theory and development of context for generation of new theory with better status, is an alternative to traditional inference based on precise mathematical proof what is frequently applied in theoretical physics. We would like to accentuate by this comment that changing of theory by introduction of new assumptions perhaps, in many cases, is more appropriate than obtaining of pure mathematical consequences with the aid of a long proof. Role of long mathematical proof in description of reality will be discussed also in connection with proof with respect of reality in what follows.

The question is why we discuss the term "selforganizing reasoning". This name follows from fact that we start from a chosen theory. Then modification of this theory towards new theory follows from a context elaborated on various ways including estimation of status. Obtained sequence of theories should go towards theory corresponding to the best approximation of reality. Thereby, changing of such a final theory will be very difficult. This is a kind of a fixed point.

Cognition manifold creates a methodological environment for selforganizing reasoning towards better recognition of reality by theory. Within this theoretical environment we can create new hypotheses 
for changing of theories, estimate accordance of theories with experimental results and estimate status of theories which can be applied to defining a kind of distance from appropriate approximation of reality.

REMARK 7.1: We accentuate the term "selforganizing reasoning" since we have to do here with evolution of theories towards a theory corrsponding to a fixed point. We can consider also a kind of distance from this fixed point and expressed with the aid of status of theory.

\section{Remarks on connections between measurement theory and creative reasoning}

Mesurement theory describes our possibility in measurement of various quantities. Then, obtained results have to be interpreted with the aid of description of measurement applied. The most known result of measurement theory within theoretical physics is represented by Heisenberg uncertainty principle.

We encounter frequently in theoretical physics very strong connection between creation of hypotheses and the uncertainty principle. Frequently this principle is applied to proof of various theorems or justification of new hypotheses. In case of uncertainty principle we manipulate various type waves for measuremet of quantities. In other words, in theoretical physics, we have to do with constraints imposed on thinking following from the uncertainty principle.

Let us note however that our measurement depends on current technical capabilities. On the other hand new hypotheses can be related to reality which perhaps is not accesssible for our measuremet directly. Perhaps our possiblities in measurement in a future can be based on entirely new phenomena and can be much more precise. Let us mention in this case properties of neutrina described within vacuum medium mechanics [17]. Neutrino described within vacuum medium mechanics has no wave function. This follows from considerably larger system of fundamental notions than that one applied to formulation of the quantum mechanics. Thereby uncertainty principle should not be taken into account in interactions of neutrina with other elementary particles. As a result of this we come to various conclusions on radius of various charged elementary particles when we apply scattering experiments of these particles with neutrina or scattering with electrons for instance.

It seems that measurement theory should be devoted to description of a system of interacting objects. One of them represents measurement apparatus which has various states and we are able to manipulate part of these states. The second one is a system which has also various states. These states can be measured quantitatively when both objects interact and we can decipher corresponding values owing to description which is at our disposal.

We see in this place that our measurement depends on description of corresponding interacting objects. We can obtain relation between various states of this system. Let it be $\mathcal{R}_{M}\left(Z_{1}, \ldots, Z_{N}\right)$, where $Z_{1}, \ldots, Z_{N}$ are quantities which can be measured. The relation $\mathcal{R}_{M}$ determines a set to which quantities $Z_{1}, \ldots, Z_{N}$ belong.

Let us notice that we can introduce to description of our system new quality associated with new hypotheses where we apply quantities which cannot be measured at this moment. This can mean that we are able to indicate a subsystem within $\mathcal{R}_{M}\left(Z_{1}, \ldots, Z_{N}\right)$. However we are not able to manipulate new introduced variables. Thereby precission of our measurement is determinad as previously by the relation $\mathcal{R}_{M}\left(Z_{1}, \ldots, Z_{N}\right)$. Our technical possibilities are not improved. However we come to the conclusion that values $Z_{1}, \ldots, Z_{N}$ which appear within the set $\mathcal{R}_{M}\left(Z_{1}, \ldots, Z_{N}\right)$ do not take all admissible values which are present there. It means that we cannot infer directly from measurement theory which values can be attained by real states. Consequently we come to the following remark:

REMARK 8.1: Measurement theory should not create constraints on creation of new hypotheses related to reality or in other words on our reasoning. Measuremet theory should be cosidered as separate theory which allow us estimate to what degree we are able to measure various quantities in relation to recognized reality. Consequently this theory can be helpful in identification of constants 
which appear in theories. However, application to inferring in direction to new description is not appropriate since such an inferring should not depend on our current technical capabilities for measurement.

\section{Remarks on reductionism and emergence}

Reductionism and emergence are notions which are frequently applied in philosophy of physics and philosophy in general [12], [13]. In particular one estimates that contemporary theoretical physics has success based on methodological reductionism. It means the attempt to reduce explanations to smaller constituents and to explain phenomena completely in terms of interactions between fundamental entities [13]. Theoretical reductionism is considered within the methodological reductionism, wherein one theory with limited predictive power can be obtained as a limiting case of another theory [13].

Emergence is understood as involving new, sometimes novel properties of a whole that are not shared by its isolated parts [13]. Problems of emergence in physics are discussed frequently in literature. Let us mention [14] for instance. One suggests that complexity leads sometimes to entirely new quality which cannot be derived form more elementary level.

It seems that both reductionism and emergence are notions which are not entirely clear. It follows from fact that they are placed on border of formal cognition. Within this work we do efforts towards formalization of cognition process as far as it would be possible. Therefore some comments on reductionism and emergence are justified.

By this paper we promote reductionistic approach to theoretical physics including also description of complex systems. This is manifested especially by concept of construction of fundamental theory. We asssume namely necessity of determination of form of space considered as a medium. It means that we wuold like to have at our disposal states of this medium expressed with the aid of variables.

Introduction of corresponding variables is associated with determination of set of fundamental notions where we estimate status. Attaining high status of assumptions is easier in this place since we have to do with simpler objects. Summarizing promotion of reductionism is expressed here by starting first from determination of variables. Then, relations appear as next stage of modelling what is manifested by notion of cognition manifold.

Emergence in this context should be understood as construction of physical theory starting from relations at first stage, neglecting at this moment determination of precise role of corresponding variables at the fundamental level. In particular corresponding relations can use variables related to larger scale. This approach is justified in complex systems. We observe some properties and try to describe them directly by relations. As a result of this we obtain a theory. However this theory is usually not fundamental. Furtheremore, we should not expect that such a theory will have sufficiently large range of validity.

Let us note that cognition process can lead to situation when it is difficult to introduce new variables directly on the most fundamental level. It would be the case when our modelling of reality is advanced and new concepts are difficult to obtaining however some relations could be observed and introduced into description as difficult to understanding. We can accept such a situation temporarily.

It seems that cognition process should be continued in spite of all towards finding fundamental assumptions as far as it is possible and constructing of theory with the aid of these assumptions. Perhaps we attain then new situation when we would have to do with necessity of considering very large number of assumptions. Perhaps we would be forced in this case to decipher chaotic phenomena for instance.

Efforts of this kind are done in [11] where the term precursor of evolutionary property is introduced. The term precursor of evolutionary property means processes in molecular system, described by fundamental laws and involved in biological evolution process, which are responsible for appearing of this particular property when biological evolution attains an assumed level of complexity where the property can be manifested.

Finding of precursors is task associated with creation of hypotheses related to fundamental level in 
order to modify the fundamental theory. It means that the term precursor manifests using additional context following from more complex processes, for obtaining new premises for making assumptions. In this case more complex processes are considered as experiments which have additional quality in comparison with experiments related to the simplest processes.

CONCLUSION 9.1: Emergence is understood here as generation of theories by construction of relations based on observed properties. Corresponding relations can have variables which represnt various scale states. These states can be difficult to understanding. One suggests to consider corresponding relations as placed in zone represented by image of a mapping $\tilde{P}_{I_{k}}$ related to a smaller scale. Then $\tilde{P}_{I_{k}}^{-1}$ represents area where pressure towards deciphering fundamental assumptions can be generated. This in turn could lead to obtaining finally theoretical speculations within structure corresponding to cognition manifold.

This kind of pressure on development of theories can create premises and hypotheses leading to perhaps unexpected consequences. Introduction of precursors manifest just this approach. It is also admissible that we attain the stage of description where further recognition of reality will not be possible.

Let us comment here role of mappings $\tilde{P}_{I_{k}}$. They are introduced in discussion on inductive reasoning. When this induction is related to diminishing of scale then we have to do with mapping which represents averaging procedure. Such an approach is elaborated in case when we consider formalization of scale of averaging applied to modelling of physical reality [5], [10]. Then theoretical reductionism is not necessarily associated with obtaining another more general theory which leads, as a limiting case, to that previous one.

We apply averaging procedure going from theory having entirely new set of assumptions and variables not directly related to previous one which is modified. It means that we admit the case when we do a jump to entirely new assumptions without modification of previous theory. Then range of validity of previous theory becomes smaller and evolution of theory is not seen as continuous process. We express this possibility by considering various elements of basis of cognition manifold. Above comments extend in fact notion of theoretical reductionism and provide to it new context.

We have to do in theoretical physics with tendency to modification of existing theories in a continuous way. We understand by this modification of separate assumptions or adding new ones to existing assumptions within modified theory. This tendency is justified by fact that physicists try to maintain hitherto attained success in modelling of obtained experimental results. However, jump to new set of assumptions gives better chance for introduction of new qualty. Let us mention concept of vacuum medium mechanics [15], [10] which is a jump from widely accepted quantum theories towards entirely new set of assumptions. As a result of this entirely new quality appears. This new quality is manifested in description of internal structure of proton as composed of three positrons [9], [10] or possibility of expression of mechanism of biological evolution on the most fundamental elementary particle level [10].

Emergence is seen within our approach as incapacity in our reasoning which we frequently have to accept. Therefore one suggests to give pressure towards changing this situation in accordance with Conclusion 9.1.

\section{On proof with respect to reality}

We use in theoretical physics mathematical theories which are designed to description of reality. Then, within corresponding theories we can carry out proof and by this we create theorems. The question is whether we are able to estimate status of theorems. In other words we should try to distinguish mathematical proof from proof with respect to reality.

Mathematical proof is understood in the same way as this is done in pure mathematics. However, each step of proof is associated with an action of dynamical system for recognition of reality. It means that increasing length of proof should lead to more complex action of dynamical system and therefore 
approximation of reality becomes then less direct. Consequently status of obtained consequence should be lowered.

In order to estimate status of consequence we should discuss status of assumptions applied to proof. Let us note that in [1] one discusses properties of equivalenc laws frequently applied in theoretical physics with respect to resolution of seeing of reality by theory. We have obtained there the following remark:

REMARK 10.1: Equivalence laws applied in physics reduce number of degree of freedom in theoretical description of reality. This follows from the fact that corresponding to this situation mapping which joins theoretical description with reality becomes then less similar to one-to-one mapping. It means that approximation of reality by theory is worse in case of application of equivalence laws what means lowering status of this description.

This remark provides an example of assumption which can be applied in a proof. When this is the case it makes status of a consequence lower. Taking into account previously discussed creteria for estimation of status of assumptions we should formulate the following remark in relation to status of consequences obtained with the aid of a mathematical proof:

REMARK 10.2: Status of a consequence obtained with the aid of mathematical proof within a physical theory should be lower than the lowest status of assumption considered in set of assumptions applied to obtaining of this consequence. Furtheremore, the larger number of assumptions applied in various steps of proof the lower status of the consequence. It means also that longer proof leads to worse approximation of reality by obtained consequence.

Above discussion follows from fact that we try to formalize cognition process. Thereby a new quality in reasoning appears. Now we have to do with proofs with respect to reality as additional aspect of theoretical physics in comparison with mathematical proof hitherto applied.

Proof with respect to reality means that we carry our usual mathematical proof which is accompanied by additional process of estimation of status of each step of the proof. In case when status of consequence is very low we have to interpret this fact as premise for modification of assumptions of whole theory in order to obtain better description of reality.

Proof with respect to reality provides additional aspect of estimation of content of physical theory. Let us consider a set of assumptions $T A$. Then we can attain set of all consequences $C(T A)$ starting from the set $T A$ by carrying out pure mathematical proofs in order to obtain the theory $T h=C(T A)$. However, taking into account proofs with respect to reality we admit a subset $C_{P R}$ of all consequences $C(T A)$ only. This means that our theory describing reality $T h_{P R}$ is smaller than $T h$.

In order to make theory $T h_{P R}$ larger we should introduce new hypotheses. All this elucidates various aspects of methodology of construction of cognition manifold as well as methodology of obtaining cross-section within cognition manifold.

\section{Examples of proof with respect to reality}

\subsection{On status of existence of virtual particles}

Let us carry out an example of proof with respect to reality. We encounter in theoretical physics concept of virtual particles. Consequently we should estimate status for existence of such particles.

We can distinguish two aspects of existence of virtual particles. The first one is related to all assumptions which create environment for this concept. The second one is related to direct reasoning leading to justification of virtual particles.

Assumptions which create environment for this concept are associated with the Dirac equation. Let us discuss content of the Dirac equation with respect to its ability to description of reality. We 
consider in this equation point-like particle which has charge and mass by assumption. Furthermore we accept wave particle duality what means that evolution of wave function entirely describes motion of this particle. The last aspect makes lower status of this description in accordance with Remark 10.1. Acceptance of wave particle duality means that we have decided to diminish resolution of our thinking to level where particles and waves are not distinguishable.

At this moment we are aware what degree of averaging of physical effects is assumed. Point-like particle means that all effects are averaged on whole particle. No internal dynamics is considered and no mechanisms of motion is introduced. Having such assumptions we are aware at this moment that resolution of this description is above internal properties of particle.

Derivation of discussed equation is based on quadratic relativistic relation between energy, mass and momentum as well as on acceptance of probabilistic interpretation of quantum mechanics.

The quadratic relativistic relation leads to situation when particles and antiparticles appear owing to taking roots of quadratic equation. Thereby, existence of particle-antiparticle pair is considered as assumption rather than consequence of this equation. In case when we would apply power of fourth order for relativistic relation between energy, mass and momentum then we have to do with four type particles.

Let us note that acceptance of probabilistic interpretation and introduction by this the probabilistic density also means that resolution of this theory is rather low and takes the level similar to level of measurability.

Joining of probabilistic density and quadratic relativistic relation is in fact risky. We impose stochasticity on perhaps unknown number of particles (particle-antiparticle system) with various properties including their interactions. At this moment we have no premises on such interactions. Then, we can expect difficulties with merging of two theoretical aspects leading to unknown and also perhaps unwanted effects. In other words we can have difficulties with interpretation of details related to reality by the Dirac equation. The Klein paradox can be interpreted just us such a dificulty.

Let us note that appearing of spin in the Dirac equation is rather strange. This follows from fact that no internal structure of particle or internal mechanisms of motion are assumed in accordance with above discussion. We can interpret this fact as introduction of spin in a phenomenological way by extra assumptions related to number of terms in development of the probability density expression. Freedom in this approach rests on fact that in case of application of larger number of terms we could obtain additional spins within this interpretation. Furtheremore no justification is introduced on form of this development. See Remark 2.3 on status of assumptions introduced by development into series.

This in turn means that no serious efforts have been done in this place in order to describe such a really important phenomenon as spin. This also means that there is no chance for detailed description of spin phenomenon within the Dirac equation without essential extension of this approach.

At this moment we should decide whether we accept the Dirac equation as such or we should improve or better justify them. We have to do in theoretical physics with the second way. The Klein paradox leads to the conclusion that the Dirac equation can be treated in fact as a multiparticle equation, where virtual particles could appear and decay during a short time. This in turn justifies application of the quanum field theory description.

Summarizing status of the Dirac equation is rather low taking into account all assumptions applied in derivation of this equation. It does not mean that many experimental results should not fit quantitatively to predictions obtained by the Dirac equation. Accordance with large set of experimental results takes place since the Dirac equation provides large number of degree of freedom and by this allows us to fit this equation to reality in an area. However we should expect difficulties when we extend range of validity of such a theory. Indeed we have to do in theoretical physics with difficulties with spin of proton for instance. It means that when we apply the Dirac equation which describes just spin to this reality then it loses in this new environment whole quality. It justifies just low status of the Dirac equation.

Formally, existence of virtual particles is based on two steps. The first one applies indeterminacy principle which states that energy fluctuation can be considerable in sufficiently short time. This step 
is in fact not admissible. We cannot infer that energy can have arbitrary value in sufficiently short time since this consequence is obtained by means of measurement theory which should not be applied to reasoning of this kind in accordance with previous discussion and Remark 8.1. Thereby this step of proof has very low status.

The second step is based on equivalence of mass and energy. This assumption allows us to change the fluctuating energy into massive virtual particles. However application of equivalence law means that this step of proof has low status in accordance with Remark 10.1.

Application of two successive steps of proof with very low status means that corresponding consequence has very low status. If we take into account additionally that assumptions which generate environment for concept of virtual particles have also not too high statatus then we come to the conclusion that status of existence of virtual particles is so low that we should entirely reject this concept.

\subsection{On status of charge conservation law}

In elementary particle physics we apply charge conservation law as law with rather high status. This status follows from accessible observations where this law is always satisfied. Our discussion in introduction suggests that we can discuss status of charge conservation law taking into account fitting of theory to reality based on experimental results and also additionally on methodology following from formalization of cognition process.

Let us discuss first status of notion of charge. This notion appears as a result of observations of electric field in vicinity of systems of charged particles interpreted within properties of Coulomb field. It has been noticed than an elementary state of electric field around single particle such as electron for instance can be seen as Coulomb field with constant $e$. This constant is interpreted as elementary charge.

Let us remark that notion of charge appears as a result of considerations related to state of environment of elementary particle and as such is assigned to elementary particle only. Considered elementary particle is seen as point-like particle. Consequently notion of charge does not represent deeper properties of elementary particle. We would say in accordance with Remark 2.1 that this notion is not interpreted as related to maximum engagement by action of the dynamical system and its sensory system in order to obtain results which next can be interpreted as the simplest information by neural network system.

We have to do in this case with rather low engagement by action of the dynamical system which represents our brain and experimental systems. This is so since experiments are relatively simple and theoretical description is also not too advanced especially in relation to smaller scales.

We can state that notion of charge is to some degree accidental and is asigned then to point-like particles. This notion is related to relatively large scale applied in modelling since analysis of electric field of elementary particles is carried out on larger distances from these particles.

Above analysis of status of notion of charge suggests that status of charge conservation law is perhaps low especially in relation to smaller scales.

We can also observe that charge conservation law can be seen as emergent property in system of elementary particles. Such a point of view is justified by fact that element of basis of cognition manifold corresponding to theoretical environment where this relation appears, is not determined sufficiently well. We have to do in this case with vacuum which is determined rather as geometrical space not interpreted directly as a medium. Various states are present in this geometrical space however without direct relation to properties of the medium and scale of averaging applied in modelling of processes in such a space is not formalized. Consequently we have difficulties with determination of mappings $\tilde{P}_{I_{k}}^{-1}$ in accordance with Conclusion 9.1 and deciphering fundamental states related perhaps to smaller scale.

The question is whether we could determine a fundamental notion with higher status than notion of charge which would be more appropriate for expression and discussion of charge conservation law. 
We have discovered creation of pair of electron and positron by application of complex experimental systems what can be interpreted as "maximum engagement by action of the dynamical system". The simplest interpretation within our neuron network system states than some components are separated. Observation of annihilation of electron and positron suggests that some components are joined again in order to recover state of vacuum medium before creation of this pair. The simplest interpretation means that we do not understand particular properties of these components and we state existence of them only.

Consequently concept that our vacuum can be interpreted as multicomponent vacuum medium has high status in accordance with Remark 2.1. Separation of components is induced predominantly by electric field. Such point of view is justified by fact that electron and positron generate static electric field. Let us assume that one exists a critical value of intensity of electric field which is necessary for separation of components. Introduced assumption can be seen as direct consequence of existence of components. Thereby it is introduced by direct and short proof what means, in accordance with the Remark 2.2, that status of such an assumption is high.

Summarizing we introduce as fundamental assumption within vacuum medium mechanics [15], [18] the following relation

$$
\mathbf{u}(\mathbf{X})=\mathbf{u}^{*}, \quad \mathbf{X} \in S_{a}
$$

where $\mathbf{u}(\mathbf{X})$ represents electric field intensity for points $\mathbf{X}$ which belong to surface $S_{a}$ of electron described within vacuum medium mechanics and $\mathbf{u}^{*}$ is vector which has length corresponding to the largest value of this quantity admissible within the vacuum medium. Exceeding of this value induces separation of components. Electron is seen within vacuum medium mechanics as extended particle. Thereby on whole surface of this extended particles we have to do with the condition (28).

Consequently external electric field of elementary particle is determined by the condition (28) which has high status. Furtheremore this condition is appropriately placed within whole theory by determination of basis of element of cognition manifold defined as four component vacuum medium [15] with well defined states. Scale of averaging applied in modelling is smaller than size of elementary particle. Therefore elementary particles are then extended objects with surfaces considered as a border between separated component and equilibrium vacuum medium.

Within context provided by vacuum medium mechanics charge conservation law can be derived. We can notice that this law can be violated when several charged particles can be so close that surface of whole such a system can be interpreted as one separation surface. Behavior of electric field in vicinity of such a surface is discussed in [18]. Such a field is flat at small distances from elementary particle and becomes Coulomb type field at larger distances. This property follows from the condition (28) and properties of components of vacuum medium.

We can state that charge conservation law can have low status especially for smaller scales. In particular within vacuum medium mechanics one assumes that proton has three-positron structure. Consequently disintegration of proton should be continued with breaking of charge conservation law. Status of such a model of proton is high and is discussed in [19], [9], [10].

\subsection{On status of existence of quarks}

Existence of quarks is inferred from experments where various symmetries are observed in elementary particles. Furtheremore one observes within experiments of scattering of electrons on protons and also neutrina on protons that proton has structure with three centers. These canters has been named partons and next identified with quarks.

Symmetries associated with properties of various type hadrons are determined by Gell-Mann Nishyijima relation

$$
Q=e\left(I_{3}+\frac{1}{2}(B+s+c+b+t)\right),
$$


where $Q$ is charge, e electron charge, $I_{3}$ is isospin, $B$ is barion number, and $s, c, b, t$ are addiitional numbers called strange, charm, bottom and top.

Consequently we should estimate status of this relation as starting point for inferring existence of quarks. Let us notice that this relation is in similar situation as charge conservation law. It happens rather as emergent property since status of element of basis within cognition manifold is not sufficiently well determined. We have to do here with space introduced by geometry. Sometimes one suggests that this is a medium. Let us mention interpretation electron or positron by the Dirac equation as holes what suggests that vacuum is a kind of medium. On the other hand one considers fluctuation of vacuum. However the last concept is associated with existence of virtual particles. Existence of virtual particles should be rejected in accordance with previous discussion since they have very low status.

Summarizing determination of element of basis of cognition manifold as a medium which has well defined states is not clear as theoretical environment for the relation (29). Then we have to consider the relation (29) as emergent property and try to extend context for this relation taking into account mappings $\tilde{P}_{I_{k}}^{-1}$ in accordance with Conclusion 9.1.

Let us note that scale of averaging applied to modelling in relation to formula (29) is rather large. We have to do with point-like particles what means that averaging is carried out over whole particle and charge conservation law is maintained. Furtheremore the relation (29) is satisfied only approximately. We encounter deviation of masses of particles from masses predicted by corresponding symmetries. Summarizing status of the relation (29) seems sufficiently large for larger scales above size of elementary particles. However problem with masses of particles suggests that status of this relation as fundamental assumption is considerably lowered.

Inferring on existence of quarks is based on rather long proof. First we should find representations of corresponding symmetry group in relation to existence of potentials. Next we have to find generators of corresponding symmetry group.

Let us also note that this inferring is continued towards lower scales. Indeed quarks have to be constituents of proton for instance. However the relation (29) considers point-like particles. It means that we should extend context by introduction of mappings $\tilde{P}_{I_{k}}^{-1}$ towards still smaller scale. Then however maintaining of charge conservation law is difficult in acordance with discussion from previous subsection. Necessity of assignation of fractional charges for quarks is important manifestation of these difficulties.

Summarizing status of existence of quarks is rather low owing to not too large status of the formula (29) in context related to various scales. Furtheremore this relation is satisfied approximately only even for scale where it is directly formulated owing to not precisely fitting masses. Quarks appear as rather long proof continued towards lower scale where formal description is in fact not constructed what additionally lowers status of their existence.

\subsection{On status of existence of bounded state of charged particle and neutrino}

Existence of bounded state of charged particle and neutrino is not considered within current theoretical physics represented by the standard model. However we observe that muon $\mu^{-}$decays into electron and two neutrina. The meson $\pi^{-}$decays into electron and three neutrina. Lepton $\tau^{-}$decays into electron and four neutrina. Therefore it is difficult to avoid the question on better description of interactions of charged particles and neutrina.

This question is difficult to discussion since system of fundamental notions of the standard model is rather poor. In particular owing to application of wave particle duality elementary particles are represented within the standard model by waves. This leads in turn to avoidance of defining of elementary particles. In order to improve this situation vacuum medium mechanics is formulated on basis of larger set of fundamental notions [15], [6], [7], [8], [9], [16], [17], [18], [19].

In the Subsection 11.2 we have estimated status of existence of components of electron and positron as high. These components, when they are joined, create stable vacuum medium. Then separation of components of vacuum medium leads to creation of electron and positron as stable particles. However we can admit also additional method of creation of particles. Part of vacuum medium considered 
as medium similar to solids can be forced to rotate without any separation of components. Such a rotation is possible since elementary particle is not point-like. Then elementary particle of this kind identified here with neutrino has no charge. Neutrino is in this case an extended elementary particle with border represented by discontinuity surface between rotating and stable vacuum medium. Model of neutrino within vacuum medium mechanics is discussed in [17].

Let us note that above considerations are simple and direct consequence of assumption that we have to do with multicomponent vacuum medium similar to solids. This assumption has high status what leads also to high status of neutrina introduced on this way.

When neutrino rotates near charged particle then static electric field of the charged particle is disturbed owing to this dynamics. This is so since propagating electric field within resting vacuum edium encounters rotating vacuum medium and gains by this its own dynamics. Thereby it is natural to expect that electromagnetic force can appear between charged particle and neutrino at small distances what can lead to unstable bounded state of these particles. More detailed description of this phenomenon is given in [17].

Thereby we have obtained possibility of existence of corresponding bounded state as direct and short proof. It means that such a possibility has sufficiently high status. Let us note that we have to do with muon $\mu^{-}$having two neutrina and mass $M_{2}=105 \mathrm{MeV}$ in accordance with its dacay, meson $\pi^{-}$with three neutrina and mass $M_{3}=139 \mathrm{MeV}$, lepton $\tau^{-}$having four neutrina and mass $M_{4}=1776 \mathrm{MeV}$. On the other hand free electron has mass $M_{0}=0.5 \mathrm{MeV}$. It means that we have not at our disposal bonded electron and one neutrino. Masses of corresponding particles increase with increasing number of neutrina. This is justified by mechanism of motion of extended particle within vacuum medium emchanics [9]. Increasing number of neutrina shields more and more efficiently external electric field and by this makes acceleration of electron more difficult. It means that we predict existence of elementary particle representing bounded state of electron and neutrino with mass $M_{1}$ placed between $M_{0}=0.5 \mathrm{MeV}$ and $M_{2}=105 \mathrm{MeV}$.

Let us note that in papers [20] and [21] physicists have reported discovery of a new boson $E(38)$ with mass $38 \mathrm{MeV}$ which is not predicted by the standard model. Such a mass fits to our prediction. Therefore we could suppose that it could be electron with one neutrino.

Summarizing existence of bounded state of electron and neutrino is obtained by reasoning based on high status asumptions and direct and short proof. Furtheremore experimental confirmation of existence of boson $E(38)$ additionally increases status of this point of view. All this suggests that unstable elementary particles should be composed of charged particles and neutrina [9] and this point of view has high status.

REMARK 11.1: Discovery of boson $E(38)$ is important manifestation of efficiency of presented here methodology based on formalization of cognition process. Prediction is carried out within vacuum medium mechanics for qualitative mathematical model of neutrina. Importance of this manifestation rests on fact that other fundamental physical theories do not predict this particle.

Above discussion is aimed at accentuating fact that discussed in this paper methodology of recognition of reality leads to qualitative theories which are predictive. Therefore methodology based on formalization of cognition process can lead to considerable advances in theoretical physics.

\section{Final remarks}

\subsection{General summary}

Formalization of cognition process is introduced here by several components. Let us mention them as follows:

1. Introduction of the term "status" of assumptions and theories. This is consequence of considering in [1] implication as associated with action of dynamical system in which mathematics is generated. 
2. Formulation of notion of the cognition manifold which is aimed at introduction of methodological environment for generation of new theories describing physical reality.

3. Promotion of selforganizing reasoning based on development of context which contains fighting about recognition of premises from various sources, as appropriate for theoretical speculations and approaching by this to better recognition of reality.

Development of theoretical description can be continued by increasing of number $s$ in creation of models $m_{\phi_{k} M_{s}}$ for a given element of basis $\mathcal{B}$ within cognition manifold. Such a development is associated with speculations on the same level of understanding of our space by adding new assumptions and hypotheses related to evolution of processes. However we can also modify our understanding of space by inductive reasoning creating new elements of basis $\mathcal{B}$. We can go for instance towards smaller scale models of our space considered as a medium. Then by means of mapping $P_{\text {In }}$ we can explain postulated models $m_{\phi_{k} M_{s}}$ related to previous element of basis. Option of way of development depends on our possibility of recognition of new premises. In the first case we have to do with simpler speculations. Transition to new element of basis $\mathcal{B}$ needs usually more deep concepts and is usually more complicated. Cooperation of both ways simultaneously can considerably develop contex related to modelling of reality.

Let us mention that cognition manifold should be applied also to gathering of information on previously introduced theories which become less important. Such a history supports development of context for new theories. In particular with the aid of obtained context we can select more optimal paths for continuation of development since we need not repeat the same ways.

The question is where from such a large necessity for theoretical speculations follows. It seems that in current theoretical physics we tend predominantly to physics associated with higher and higher energy. This is manifested by various facilities which are able to carry out experiments for high energy physics. However, low energy physics is very important. Let us mention physical foundations for theoretical biology or nanotechnology. In the paper [10] one indicates important role of attractor defined at the elementary particle level and governing selforganization manifested at the molecular level. This attractor is responsible for precision of nanotechnological processes as well as for biological evolution. Thereby we are able to express mechanism of biological evolution on the most fundamental elementary particle level.

Foundations for mechanism of this selforganization can be associated with high energy physics and low energy physics. Explanation of corresponding selforganization can be based on theoretical speculations and needs more subtle experimental results related to low energy physics. Experiments related to various states of vacuum medium associated with elementary particles are not carried out yet. Let us mention here for instance experimental investigations related to evolution of components responsible for state of corresponding wave function. Such experiments are difficult since theory and experiment are strongly conjugated for more subtle processes. Therefore they need more clear theoretical foundations frequently obtained with the aid of various hypotheses. We can expect that perhaps larger set of experiments can be necessary for extraction from them confirmation of formulated previously hypotheses. Then theoretical description has to be sufficiently complex in order to see this fine reality.

The title of this paper accentuates role of speculations in theoretical physics. We come to the conclusion that role of theory increases when we develop investigations towards more fundamental level. Indeed experiments are then less clear and need interpretation by theories which use hypotheses. All this elucidates the problem what is important in development of current theoretical physics.

The term qualitative theory is considered frequently as equivalent to preliminary stage of development of theory. Then, any theory which provides quantitative results is seen as more serious and advanced. However such a point of view is now not sufficienly justified. We should decide what is more important quantitative theory which is not universal or qualitative theory which can be universal in a future.

The first theory can be applied in some range of validity. The second theory gives us a hope for 
description of all observed phenomena and perhaps to predict some new ones. Furtheremore transition with time from qualitative to quantitative theory should be relatively easy. This step needs determination of constants which are provided by qualitative models only. Thereby, main efforts for construction of theory with large range of validity are done within qualitative speculations where we have to track down premises for making assumptions. This task seems to be the most difficult when we construct fundamental theory. Qualitative theory can be complex since it can be based on large number of equivalece classes corresponding to various partial phenomena. Let us also note that estimation of status of asumptions introduced can be difficult. Consequently it seems that the second aim related to formulation of qualitative theory is much more important for development of theoretical physics. We come to the following conclusion:

CONCLUSION 12.1: Construction of qualititative theory on way towards universal theory is much more important for development of theoretical physics than quantitative theories which fit to experimental results in some range of validity only. Therefore the main task for theoretical physics is to create activity by systematic speculations considered as method of recognition of reality. The term "systematic" means that speculations should be well placed within cognition manifold and selforganizing reasoning. Thereby the term qualitative theory can be associated with advanced theory and should not be identified with preliminary stage of development.

This paper expresses tendency towards formalization of cognition process as additional aspect of modelling in comparison with application of precise mathematics to description of reality only. In theoretical physics the cognition process is not formalized yet. Premises for application of mathematics follow in current theoretical physics from philosophy of physics and philosophy of sciences in general. This means that within this paper partial formalization of some aspects of philosophy of physics is carried out. I suggest necessity of shifting this formalization as far as it is possible ahead. This in turn should be helpful in determination of resolution of seeing of reality by theory, thereby also by our brain, so precisely as it would be possible.

Consequently one suggests to carry out formalization of cognition process in order to obtain better comprehension of theoretical description in relation to reality. It seems that space for more systematic theoretical speculations should be enlarged maintaining discipline as far as it is possible just owing to introduced formalization.

\subsection{New possibilities for development of theoretical physics}

Let us remark that elaborated here methodology was applied to formulation of vacuum medium mechanics. This creates new situation for development of theoretical physics. Vacuum medium mechanics sees reality within current set of experimental results which differs from reality described by other fundamental theories including the standard model.

The vacuum medium mechanics sees proton as composed of three positrons [19], [9]. Neutrina can create unstable bounded states with charged particles [17] what changes point of view on weak interactions. Now such interactions have electromagnetic character induced by rotation of neutrino within static electric field of charged particle. All this leads to entirely new classification of elementary particles [9]. There are no quarks. Boson $\mathrm{Z}$ is not carrier of electroweak interactions in direct interactions between electron and neutrino. Big Bang model is considered as explosion of space on the inside of giant black hole [16] and leads to processes which are in accordance with astrophysical observations. Gravitational field can be interpreted as phenomenon on the most fundamental level having clear influence on motion of particles. Dark energy is interpreted directly as energy of gravitational field generated by external black hole wall created after the Big Bang. Then accelerated expansion of universe is interpreted as attraction of visible matter by mass of external black hole wall and no inflation is considered. Unification of all elementary particle interactions is obtained directly.

We have also to do with entirely new quality within vacuum medium description. Annihilation of electron and positron produces electromagnetic radiation and additionally also explosion of space. 
This property is not manifested in current fundamental theories since particles are considered there predominantly as point-like. Another new quality is related to concept of chain state of particles with the same charge [6], [7] which has high status. This leads to electronic interactions which are not described by quantum theories. Consequences are serious. We are able to suggest mechanism of biological evolution considered on the most fundamental physical level [11], [10], [7]. Furtheremore such processes allow us to discuss source of precission for molecular nanotechnology [10].

New opportunity for further investigations is also associated with possibility of continuation of cognition process by considering deep structure of vacuum medium related to scale $S_{D V M}<S_{V M}$ with a hope of better understanding of mechanisms of evolution of wave function as well as better understanding of whole vacuum medium.

Let us notice that traditional point of view on development of theoretical physics suggests that we need new experiments for confirmation of various phenomena seen within vacuum medium mechanics. This situation differs from situation of the standard model where we have at our disposal large number of such experiments.

Indeed some additional experiments for vacuum medium mechanics could be useful especially in relation to disintegration of proton. Some of them are done or can be reinterpreted. Let us mention here discussion on arguments supporting model of three-positron structure of the proton [19]. Entirely new experiment gives evidence for existence of boson $\mathrm{E}(38)$ what supports directly the vacuum medium mechanics. However we encounter entirely new circumstances discussing experimental confirmation of vacuum medium mechanics. At this moment existing experments support to larger degree vacuum medium mechanics than the standard model.

Let us mention in this place dacay of pion for instance. Products of this decay suggests directly that pion is composed of electron and three neutrina. The standard model states that it is composed of two quarks what has a justification within this theory. However direct observation having larger status is removed by the standard model as less important. Similar situation happens in interpretation of neutrina. The standard model treats neutrino traditionally by wave particle duality as a wave. However no wave properies of neutrina is confirmed directly by experiment in similar way as this is done for electron for instance. It suggests directly that resolution of seeing of reality by the standard model is low and is not based on assumptions related to clear observations.

Introduction of larger system of fundamental notions within vacuum medium mechanics allows us interpret neutrino better [17], [9]. This in turn allows us to interpret decay of pion as confirmation of existence of bounded state of electron and three neutrina. Similar sitation is related to neutron, its dacay and interpretation of its composition as bounded state of proton, electron and neutrino within vacuum medium mechanics [9].

Above concise discussion states that improved methodology enables us to take over existing experimental results of physics in order to justify vacuum medium mechanics to larger degree than the standard model. We see in this place also increasing role of conjugation of theory and experimental results in interpretation of reality.

Consequently new possibilities for development of theoretical physics rest on development of various speculation lines in accordance with assumed methodology which takes into account estimation of status of assumptions and theories. Development of vacuum medium mechanics is an example of such a way. Furtheremore new possibilities for development rest also on develpment of methodology which would elucidate better essence of conjugation of theory and experimental results. Continuation of development in this direction should improve our interpretation of experimental results.

\subsection{New possibilities for development of philosophy of science}

This paper creates a new quality also for philosophy of physics. We observe in literature various attempts for elaboration of methodology for construction of physical theories, let us mention [23], [24] for instance. We observe there structuralist approach which perhaps is the most close to that one presented in this work. However we can indicate important difference. 
Structuralist approach formalizes theoretical description of reality. However methods of changing of theories in order to reflect reality better are not entirely clear. Neverhtheless some comments on this matter are done. This is so since cognition process is not formalized. As a result of this methodology discussed in [23], [24] is referred to examples of existing theories and does not provide pressure on direct development of theoretical physics.

We would say that this work provides new opportunity for development of philosophy of physics which consists in formalization of cognition process. Cognition theory is a part of philosophy of science. Thereby formalization of this process is an important task for philosophy. Let us mention that we should have attitude which force us to formalization of all what is possible. Then "metatheoretical" reasoning should be continued beyond border determined by area of this formalization. It means that we should avoid "metatheoretical" reasoning in area which could be formalized.

The term cognition is related also to cognitive psychology. On the other hand theory of knowledge called epistemology is applied in philosophy. We have to do in this paper with mixing of both terms. Cognition considered here happens owing to action of a dynamical system in an environment. The term "status of assumptions" is associated to some degree with perception. This perception happens owing to motor and sensory system of our dynamical system. However it also happens on the inside of neural network system between its subsystems [1]. This in turn can lead to interpretation of various aspects of mathematics created there.

We would say that we try to introduce point of view that mathematics is considered here as a kind of sense for recognition of reality. By direct connections of mathematics with dynamical system we obtain a hope for physical interpretation of mathematics. This could be done when the dynamical system would be derived as a physical system. Then perception by mathematics could be perhaps better explained.

Formalization of cognition process discriminates two kinds of thinking. The first one is based on information processing and is related to larger degree to pure mathematics and power of brain. The second one is based on perception and is related to fitting of mathematical description to reality and to larger degree to sensitivity of brain. The last kind of thinking is perhaps more close to philosophy of science.

Within this context we come also to the conclusion that formalization of cognition process tends to improvement of resolution of seeing of reality by theory what is manifested just on way how mathematics is applied. In particular criteria for estimation of status of assumptions and theories should lead to improvement of this resolution.

Let us also note that owing to discussed above methodology we have a hope to formalize axiology in a future. This is so since owing to vacuum medium mechanics obtained with the aid of introduced methodology we can discuss formulation of theoretical biophysics which contains mechanisms of biological evolution based on the most fundamental physical processes [11]. Such a possibility can be discussed since we are able to define an attractor within molecualr system and based on electronic processes which governs selforganization of biological molecules. This in turn gives foundations for defining a dynamical system which would realize cognition process associated with evolution on way discussed within this work. Then system of values within this dynamical system can be considered. Positive value is associated with action of this dynamical system which is in accordance with appropriate direction for evolution of a given organism. This in turn can be seen as opportunity for fundamental description of emotions as associated with created system of values.

We obtain also new situation related to general view on development of theoretical physics based on philosophy of physics. In accordance with [4] we have to do in theoretical physics with paradigms. Within paradigms evolution of theory is continued until it attains an appropriate stage. This stage of development indicates whether the theory can be removed or can become dominant as a result of comparison with other theories within other paradigms.

Paradigm approach has some deficiencies. It favors to larger degree organisation of groups of scientists. Progress in theoretical physics can be seen in such a case as of secondary importance since works within paradigms are preferred. Perhaps persons which try to introduce entirely new point of view 
will not be accepted as not fitting to current paradigms and by this to organised groups of scientists. Consequently, paradigm approach stabilizes theoretical physics on current level of development. Pressure on cognition process associated with development of theoretical physics within this approach is in fact stopped owing to lack of appropriate mechanisms leading to development beyond the paradigms. This in fact has important consequences since estimation of quality of scientific work is not associated with fighting on new quality in theoretical physics.

The question is why such a situation appears. It seems that Kuhn did not see directly how new quality in theoretical physics appears by efforts of scientific workers. Instead of this various final steps have been analysed. Let us note that just each final step has its own history which is not seen directly, and is based on way how theoretical physicist is fighting and generates by this pressure on development. In particular new results appear with the aid of attained stage of scientific development of theoretical physicist and this development can take very large part of his scientific life. Thereby efficiency of scientific development has to be seen as important part of scientific work since just this development determines starting point for fighting on new results.

Summarizing this discussion, we see that paradigm approach is not introduced with the aid of analysis of methods of work of theoretical physicists taking into account all aspects of this work. This leads also to avoiding of introduction of systems of opinions which take into account methods of scientific work and real efforts related to doing progress. All this stabilizes current stage of development of theoretical physics and in fact acts against development. By this discussion one suggests also that philosophers of physics should to lager degree analyse scientific results considered as intermediate steps which lead to final important result instead of final results only what is done predominantly by historical analysis. Such an attitude makes better understanding how real progress is made. Formalization of cognition process seems support just such an approach.

This work suggests that we could consider speculation line placed within cognition manifold instead of paradigm. Then development of new line is natural and is not in contradiction with other speculation lines. Furtheremore, even in case of rejection of a theory which is developed with the aid of speculative reasoning, corresponding speculation line is justified as separate tasks which should be finished with appropriate conclusions. All this is considerably better for scientific workers which create theories which perhaps will be rejected since they provide notwithstanding recognition of reality by extension of context with the aid of investigated speculation line.

It seems that speculation lines allow us to make better observations how development is continued by efforts of theoretical physicists and estimate amount of these efforts.

Above discussion indicates that development of context can be additional and important aim of investigations. With the aid of extended context, we could obtain perhaps new premises, which was not seen previously without this context. Struggle for obtaining of new premises is fundamental for creation of new speculation lines and estimation of status of assumptions and theories.

\section{References}

1. J. Kaczmarek, On methodology of constructing of physical theories, Journal of Pure and Applied Mathematics: Advances and Applications, 15(1), 23-60 (2016).

2. J. Kaczmarek, On emergence of intelligence in biological systems and methods of modelling of reality necessary for development of theoretical biology, Far East Journal of Applied Mathematics, 75(2) 101-150, (2013).

3. K. Popper, The Logic of Scientific Discovery, Taylor and Francis e-Library ed., (2005).

4. T.S. Kuhn, The Structure of Scientific Revolutions, University of Chicago Press, (1962).

5. J. Kaczmarek, A method of multiscale modelling considered as a way leading to unified mechanics of materials, Acta Machanica, 226, 14191443 (2015), DOI 10.1007/s00707-014-1261-7.

6. J. Kaczmarek, Concept of the chain state of particles with the same charge, Advanced Studies in Theoretical Physics, 3(1-4) 13-33, (2009).

7. J. Kaczmarek, On the role of the electronic chain state for evolution of molecular systems leading to a protocell, Advanced Studies in Theoretical Physics, 4(9), 413-435 (2010).

8. J. Kaczmarek, Surface superuidity property as a mechanism responsible for rotation of the electron, Advanced Studies in Theoretical Physics, 6(28), 1355-1369 (2012).

9. J. Kaczmarek, On structural classi_cation of elementary particles within vacuum medium mechanics, Malaysian Journal of Physics, 36(1), 1-19 (2014).

10. J. Kaczmarek, Problems Associated with Transferring of Engineering to Small Scale - Towards Theoretical Nanotechnology, Acta Physica Polonica A, 130(6), 1295-1323 (2016). 
11. J. Kaczmarek, On a general formulation of theoretical biophysics which accentuates dominant role of dynamics of processes over structures within biological systems, submitted.

12. Carl Gillett, Reduction and emergence in science and philosophy, Cambridge University Press (2016).

13. Hildegard Meyer-Ortmanns, On the Success and Limitations of Reductionism in Physics, in B. Falkenburg and M. Morrison (eds.), Why More Is Di_erent, Springer-Verlag Berlin Heidelberg (2015).

14. Joana Rigato, Looking for Emergence in Physics, Firenze University Press, (2017).

15. J. Kaczmarek, Speculative mechanics: a concept for modelling the vacuum medium, Phys. Essays, 12(4), 709-732 (1999).

16. J. Kaczmarek, Processes related to black hole state described within four-component vacuum medium model, Advanced Studies in Theoretical Physics, 3(1-4), 35-63 (2009).

17. J. Kaczmarek, A prototype model of the free neutrino, Advanced Studies in Theoretical Physics, 2(5-8), 13-33 (2008).

18. J. Kaczmarek, A model of electric_eld in the vicinity of charged particle, Advanced Studies in Theoretical Physics, 7(24), 1165-1187 (2013).

19. J. Kaczmarek, Arguments supporting model of three-positron structure of the proton, Advanced Studies in Theoretical Physics, 5(2), 63-75 (2011).

20. E.van Beveren, G. Rupp, Material evidence of a $38 \mathrm{MeV}$ boson, arXiv:1202.1739 [hep-ph].

21. Kh.U. Abraamyan, A.B. Anisimov, M.I. Baznat, K.K. Gudima, M.A. Nazarenko, S.G. Reznikov and A.S. Sorin, Observation of the E(38)-boson, arXiv, (2012).

22. W.A. Pogorzelski, Classical functional calculus, PWN Warszawa (1981).

23. W. Balzer, C.U. Moulines, J.D. Sneed, An architectonic for science. The Structuralist Program, by D.Reidel Publishing Company (1987).

24. I. Niiniluoto, Is science progressive, Springer Science+Business Media Dordrecht, (1984).

\section{ФОРМАЛІЗАЦЯ ПРОЦЕСУ ПІЗНАННЯ ЯК ДОДАТКОВОГО КОМПОНЕНТА, ВІДПОВІДАЛЬНОГО ЗА РОЗВИТОК ТЕОРЕТИЧНОЇ ФІЗИКИ Ярослав Качмарек}

Institute of Fluid-Flow Machinery, Polish Academy of Sciences 80-231 Gdansk, ul. J. Fiszera 14, Poland

Теоретична фізика досягла етапу, коли слід враховувати нові методичні підходи. Зокрема, вони повинні впроваджувати більшу дисципліну в теоретичних спекуляціях. У цій статті представлено концепцію багатозначного пізнання як методологічної основи для розвитку опису реальності теоретичною фізикою за допомогою теоретичних міркувань. Можна припустити, що це спосіб побудови фундаментальних і універсальних фізичних теорій. Багатозначне пізнання складається 3 основи, яка представляє моделі нашого простору як середовища, і представляє моделі процесів у відповідному просторі. Моделі засновані на доступних експериментальних результатах, а також на нових припущеннях та гіпотезах, отриманих теоретичними міркуваннями. 3 метою підтримання дисципліни в цих міркуваннях розглядається стан припущень і теорій, що охоплюються багатозначним пізнанням як функція, визначена на елементах волокон. Наголошується важливість самоорганізації міркувань як таку, що більше підходить для розпізнавання дійсності порівняно 3 точним чистим математичним доказом. Розглядається також докази по відношенні до реальності. Ця стаття розглядається як спроба формалізації процесу пізнання, яка характеризується трьома основними компонентами: багатозначним пізнанням, статусом припущень теорії та концепцією самоорганізації міркувань.

КЛЮЧОВІ СЛОВА: методологія побудови універсальних фізичних теорій, пізнавальна різноманітність, формалізація процесу пізнання 